\title{
Electron density distribution and solar plasma correction of radio signals using MGS, MEX, and VEX spacecraft navigation data and its application to planetary ephemerides *
}

\author{
A. K. Verma ${ }^{1,2}$, A. Fienga ${ }^{1,3}$, J. Laskar ${ }^{3}$, K. Issautier ${ }^{4}$, H. Manche ${ }^{3}$, and M. Gastineau ${ }^{3}$ \\ 1 Observatoire de Besançon, CNRS UMR6213, 41bis Av. de l'Observatoire, 25000 Besançon, France \\ 2 CNES, Toulouse, France \\ e-mail: ashok@obs-besancon.fr \\ 3 Astronomie et Systèmes Dynamiques, IMCCE-CNRS UMR 8028, 77 Av. Denfert-Rochereau, 75014 Paris, France \\ ${ }^{4}$ LESIA, Observatoire de Paris, CNRS, UPMC, Université Paris Diderot, 5 place Jules Janssen, 92195 Meudon, France
}

Received 25 June 2012 / Accepted 19 December 2012

\section{ABSTRACT}

\begin{abstract}
The Mars Global Surveyor (MGS), Mars Express (MEX), and Venus Express (VEX) experienced several superior solar conjunctions. These conjunctions cause severe degradations of radio signals when the line of sight between the Earth and the spacecraft passes near to the solar corona region. The primary objective of this work is to deduce a solar corona model from the spacecraft navigation data acquired at the time of solar conjunctions and to estimate its average electron density. The corrected or improved data are then used to fit the dynamical modeling of the planet motions, called planetary ephemerides. We analyzed the radio science raw data of the MGS spacecraft using the orbit determination software GINS. The range bias, obtained from GINS and provided by ESA for MEX and VEX, are then used to derive the electron density profile. These profiles are obtained for different intervals of solar distances: from $12 R_{\odot}$ to $215 R_{\odot}$ for MGS, $6 R_{\odot}$ to $152 R_{\odot}$ for MEX, and from $12 R_{\odot}$ to $154 R_{\odot}$ for VEX. They are acquired for each spacecraft individually, for ingress and egress phases separately and both phases together, for different types of solar winds (fast, slow), and for solar activity phases (minimum, maximum). We compared our results with the previous estimations that were based on in situ measurements, and on solar type III radio and radio science studies made at different phases of solar activity and at different solar wind states. Our results are consistent with estimations obtained by these different methods. Moreover, fitting the planetary ephemerides including complementary data that were corrected for the solar corona perturbations, noticeably improves the extrapolation capability of the planetary ephemerides and the estimation of the asteroids masses.
\end{abstract}

Key words. celestial mechanics - ephemerides - Sun: corona

\section{Introduction}

The solar corona and the solar wind contain primarily ionized hydrogen ions, helium ions, and electrons. These ionized particles are ejected radially from the Sun. The solar wind parameters, the velocity, and the electron density are changing with time, radial distances (outward from Sun), and the solar cycles (Schwenn \& Marsch 1990, 1991). The strongly turbulent and ionized gases within the corona severely degrade the radio wave signals that propagate between spacecraft and Earth tracking stations. These degradations cause a delay and a greater dispersion of the radio signals. The group and phase delays induced by the Sun activity are directly proportional to the total electron contents along the line of sight (LOS) and inversely proportional with the square of carrier radio wave frequency.

By analyzing spacecraft radio waves facing a solar conjunction (when the Sun directly intercepts the radio signals between the spacecraft and the Earth), it is possible to study the electron content and to better understand the Sun. An accurate determination of the electron density profile in the solar corona and in the solar wind is indeed essential for understanding the energy transport in collision-less plasma, which is still an open question (Cranmer 2002). Nowadays, mainly radio scintillation and

$\star$ Tables 5,6 and Appendix A are available in electronic form at http://www. aanda.org
Table 1. Previous models based on in situ and radio science measurements (see text for detailed descriptions).

\begin{tabular}{lcc}
\hline \hline Spacecraft & Data type & Author \\
\hline Mariner 6 and 7 & radio science & Muhleman et al. (1977) \\
Voyager 2 & radio science & Anderson et al. (1987) \\
Ulysses & radio science & Bird et al. (1994) \\
Helios land 2 & in situ & Bougeret et al. (1984) \\
Ulysses & in situ & Issautier et al. (1998) \\
Skylab & Coronagraph & Guhathakurta et al. (1996) \\
Wind & solar radio & Leblanc et al. (1998) \\
& burst III & \\
\hline
\end{tabular}

white-light coronagraph measurements can provide an estimation of the electron density profile in the corona (Guhathakurta \& Holzer 1994; Bird et al. 1994; Guhathakurta et al. 1996, 1999; Woo \& Habbal 1999). However, the solar wind acceleration and the corona heating take place between 1 to $10 R_{\odot}$ where in situ observations are not possible. Several density profiles of solar corona model based on different types of data are described in the literature (Table 1). The two viable methods that are generally used to derive these profiles are (Muhleman \& Anderson 1981) (1) direct in situ measurements of the electron density, speed, and energies of the electron and photons, and (2) an 

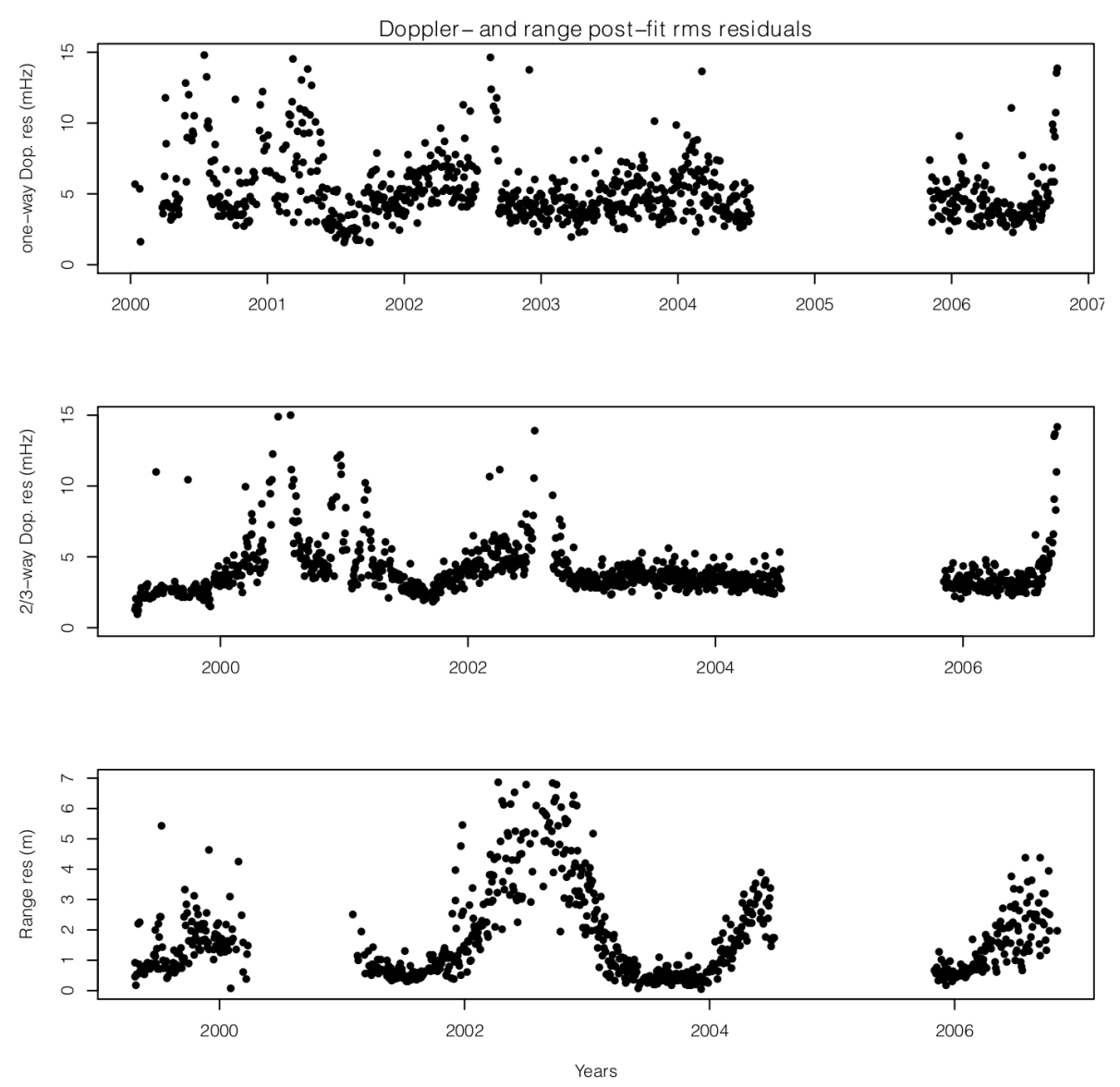

Fig. 1. Doppler- and range rms of post-fit residuals of MGS for each two day data-arc. The residuals show the accuracy of the orbit determinations. The peaks and gaps in residuals correspond to solar conjunction periods of MGS. analysis of single- and dual-frequency time delay data acquired from interplanetary spacecraft.

We performed such estimations using Mars Global Surveyor (MGS), Mars Express (MEX), and Venus Express (VEX) navigation data obtained from 2002 to 2011. These spacecraft experienced several superior solar conjunctions. This happened for MGS in 2002 (solar activity maximum), for MEX in 2006, 2008, 2010, and 2011 (solar activity minimum), and for VEX in 2006, 2008 (solar activity minimum). The influences of these conjunctions on a spacecraft orbit are severely noticed in the post-fit range and can be seen in the Doppler residuals obtained from the orbit determination software (see Fig. 1).

In Sect. 2, we use Doppler- and range-tracking observations to compute the MGS orbits. From these orbit determinations, we obtained range systematic effects induced by the planetary ephemeris uncertainties, which is also called range bias. For the MEX and VEX spacecraft, these range biases are provided by the (ESA; Fienga et al. 2009). These range biases are used in the planetary ephemerides to fit the dynamical modeling of the planet motions. For the three spacecraft, solar corona corrections were not applied in the computation of the spacecraft orbits. Neither the conjunction periods included in the computation of the planetary orbits.

In Sect. 3, we introduce the solar corona modeling and the fitting techniques that were applied to the range bias data. In Sect. 4, the results are presented and discussed. In particular, we discuss the new fitted parameters, the obtained average electron density, and the comparisons with the estimations found in the literature. The impact of these results on planetary ephemerides and new estimates of the asteroid masses are also discussed in this section. The conclusions of this work are given in Sect. 5.

\section{Data analysis of MGS, MEX, and VEX spacecraft}

\subsection{Overview of the MGS mission}

The MGS began its Mars orbit insertion on 12 September 1997. After almost sixteen months of orbit insertion, the aerobraking event converted the elliptical orbit into an almost circular two-hour polar orbit with an average altitude of $378 \mathrm{~km}$. The MGS started its mapping phase in March 1999 and lost communication with the ground station on 2 November 2006. The radio science data collected by the Deep Space Network (DSN) consist of one-way Doppler, 2/3 way ramped Doppler and twoway range observations. The radio science instrument used for these data sets consists of an ultra-stable oscillator and the normal MGS transmitter and receiver. The oscillator provides the reference frequency for the radio science experiments and operates on the X-band 7164.624 MHz uplink and 8416.368 MHz downlink frequency. Detailed information of observables and reference frequency are given in Moyer (2003).

\subsubsection{MGS data analysis with GINS}

The radio science data used for MGS are available on the National Aeronautics and Space Administration (NASA) planetary data system (PDS) Geoscience website ${ }^{1}$. These observations were analyzed with the help of the Géodésie par Intégrations Numériques Simultanées (GINS) software provided by the Centre National d'Études Spatiales (CNES). GINS numerically integrates the equations of motion and the associated variational equations. It simultaneously retrieves the physical

1 http://geo.pds.nasa.gov/missions/mgs/rsraw.html 
parameters of the force model using an iterative least-squares technique. Gravitational and non-gravitational forces acting on the spacecraft are taken into account. The representation of the MGS spacecraft macro-model and the dynamic modeling of the orbit used in the GINS software are described in Marty et al. (2009).

For the orbit computation, the simulation was performed by choosing two day data-arcs with two hours (approx. one orbital period of MGS) of overlapping period. From the overlapping period, we were then able to estimate the quality of the spacecraft orbit determination by taking orbit overlap differences between two successive data-arcs. The least-squares fit was performed on the complete set of Doppler- and range-tracking data-arcs corresponding to the orbital phase of the mission using the DE405 ephemeris (Standish 1998). To initialize the iteration, the initial position and velocity vectors of MGS were taken from the SPICE Navigation and Ancillary Information Facility (NAIF) kernels ${ }^{2}$.

The parameters that were estimated during the orbit fitting are (1) the initial position and velocity state vectors of the spacecraft, (2) the scale factors $F_{D}$ and $F_{S}$ for drag acceleration and solar radiation pressure, (3) the Doppler- and range residuals per data-arc, (4) the DSN station bias per data arc, and (5) the overall range bias per data-arc to account the geometric positions error between the Earth and the Mars.

\subsubsection{Results obtained during the orbit computation}

In Fig. 1, we plot the root mean square (rms) values of the Doppler- and range post-fit residuals estimated for each data-arc. These post-fit residuals represent the accuracy of the orbit determination. To plot realistic points, we did not consider $19 \%$ of the data-arcs during which the rms value of the post-fit Doppler residuals are above $15 \mathrm{mHz}$, the range residuals are above $7 \mathrm{~m}$, and the drag coefficients and solar radiation pressures have unrealistic values. In Fig. 1, the peaks and the gaps in the post-fit residuals correspond to solar conjunction periods. The average value of the post-fit Doppler- and two-way range residuals are less than $5 \mathrm{mHz}$ and $1 \mathrm{~m}$, which excludes the residuals at the time of solar conjunctions.

\subsection{MEX and VEX data analysis}

The MEX and VEX radiometric data were analyzed done by the European Space Agency (ESA) navigation team. These data consist of two-way Doppler- and range measurements. These data sets were used for the orbit computations of MEX and VEX. However, despite their insignificant contribution to the accuracy of the orbit computation, range measurements are mainly used for the purpose of analyzing errors in the planetary ephemerides. These computations are performed with the DE405 (Standish 1998) ephemeris. The range bias obtained from these computations is provided by ESA, and we compared them to light-time delays computed with the planetary ephemerides (Intégrateur Numérique Planétaire de l'Observatoire de Paris (INPOP)), version 10b, (Fienga et al. 2011b), and the DE421 (Folkner et al. 2008) ephemerides. For more details, see Fienga et al. (2009).

\subsubsection{MEX: orbit and its accuracy}

Mars Express is the first ESA planetary mission for Mars, launched on 2 June 2003. It was inserted into Mars orbit on 25 December 2003. The orbital period of MEX is roughly $6.72 \mathrm{~h}$

\footnotetext{
2 http://naif.jpl.nasa.gov/naif/
}

and the low polar orbit attitude ranges from $250 \mathrm{~km}$ (pericenter) to $11500 \mathrm{~km}$ (apocenter).

The MEX orbit computations were made using 5-7-day track data-arcs with an overlapping period of two days between successive arcs. The differences in the range residuals computed from overlapping periods are less than $3 \mathrm{~m}$, which represents the accuracy of the orbit determination. As described in Fienga et al. (2009), there are some factors that have limited the MEX orbit determination accuracy, such as the imperfect calibrations of thrusting and the off-loading of the accumulated angular momentum of the reaction wheels and the inaccurate modeling of solar radiation pressure forces.

\subsubsection{VEX: orbit and its accuracy}

Venus Express is the first ESA planetary mission for Venus, launched on 9 November 2005. It was inserted into Venus orbit on 11 April 2006. The average orbital period of VEX is roughly $24 \mathrm{~h}$ and its highly elliptical polar orbit attitude ranges from $185 \mathrm{~km}$ (pericenter) to $66500 \mathrm{~km}$ (apocenter). However, data were almost never acquired during the descending leg of orbit, nor around periapsis (Fienga et al. 2009).

The orbit was computed in the same manner as the MEX. The computed orbit accuracy is more degraded than MEX (Fienga et al. 2009). This can be explained by the unfavorable patterns of tracking data-arcs, the imperfect calibration of the wheel off-loadings, the inaccurate modeling of solar radiation pressure forces, and the characteristics of the orbit itself. The differences in the range residuals computed from overlapping periods are from a few meters to ten meters, even away from the solar conjunction periods.

\subsection{Solar conjunction: MGS, MEX, VEX}

The MGS, MEX, and VEX experienced several superior solar conjunctions. In June 2002, when the solar activity was maximum, the MGS Sun Earth Probe (SEP) angle (see Fig. 3) remained below $10^{\circ}$ for two months and went at minimum to $3.325^{\circ}$ according to available data. For MEX, the SEP angle remained below $10^{\circ}$ for two months and was at minimum three times: in October 2006, December 2008, and February 2011. Similarly, the VEX SEP angle remained below $8^{\circ}$ for two months and was at minimum in October 2006 and June 2008. The MEX and VEX superior conjunctions happened during solar minima. The influences of the solar plasma on radio signals during solar conjunction periods have been noticed through postfit range and Doppler residuals, obtained during the orbit computations. Owing to insufficient modeling of the solar corona perturbations within the orbit determination software, no correction was applied during the computations of the spacecraft orbit and range rate residuals. The peaks and gaps shown in Fig. 2 demonstrate the effect of the solar conjunction on the range bias. The effect of the solar plasma during the MEX and VEX conjunctions on the radiometric data are described in Fienga et al. (2009), and for the MGS it is shown in Fig. 1. The range bias (Fig. 2) during solar conjunctions is used for deriving the electron density profiles of a solar corona model. These profiles are derived separately from the orbit determination.

\section{Solar corona model}

\subsection{Model profile}

As described in Sect. 1, propagations of radio waves through the solar corona cause a travel-time delay between the Earth 

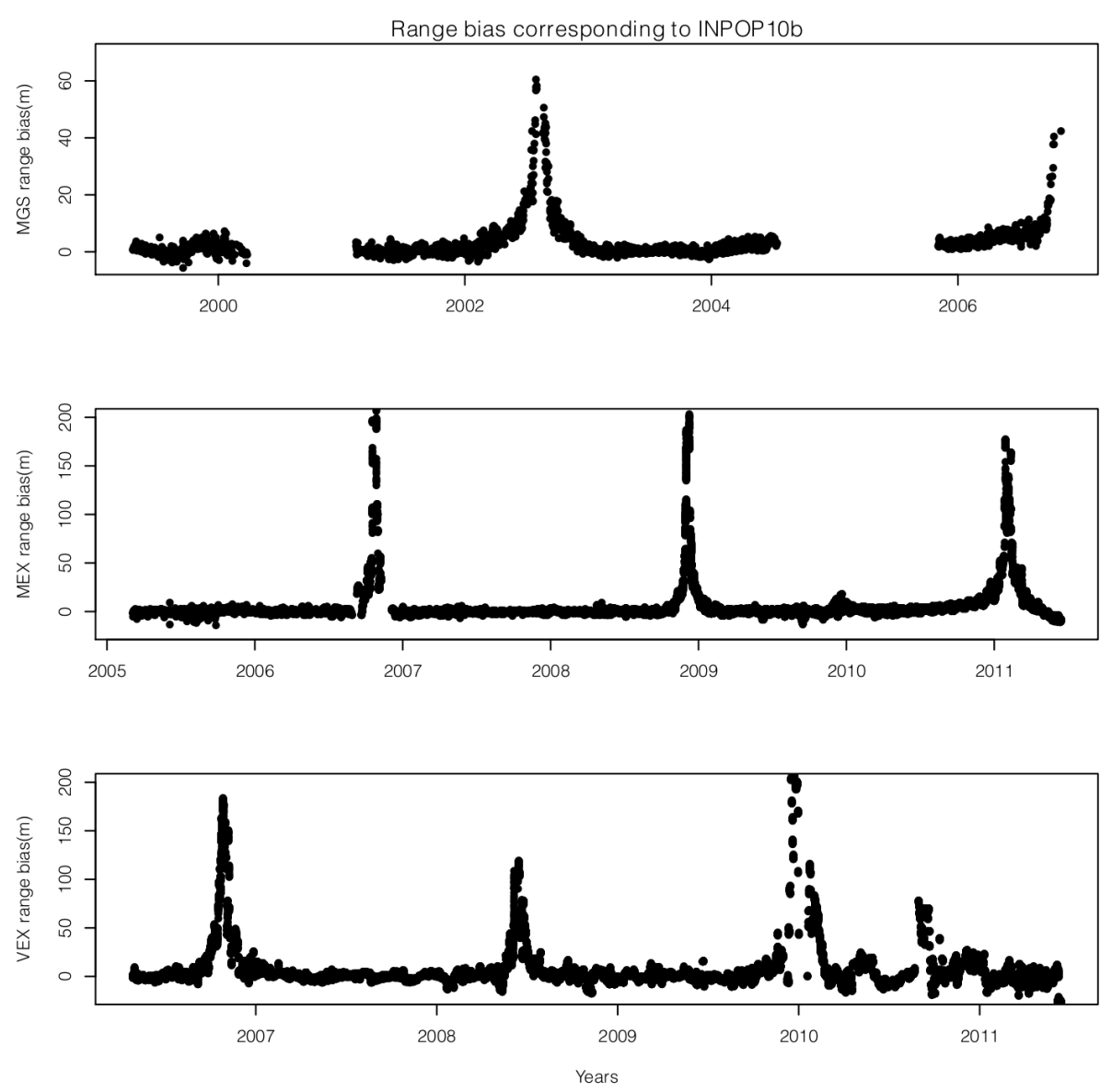

Fig. 2. Systematic error (range bias) in the Earth-Mars and the Earth-Venus distances obtained from the INPOP10b ephemeris: (top panel) range bias corresponding to the MGS obtained for each two day data-arc; (middle and bottom panels) range bias corresponding to the MEX and the VEX. station and the spacecraft. These time delays can be modeled by integrating the entire ray path (Fig. 3) from the Earth station $\left(L_{\text {Earth }_{\mathrm{s} / \mathrm{n}}}\right)$ to the spacecraft $\left(L_{\mathrm{s} / \mathrm{c}}\right)$ at a given epoch. This model is defined as

$\Delta \tau=\frac{1}{2 c n_{\text {cri }}(f)} \times \int_{L_{\mathrm{Earth}_{\mathrm{s} / \mathrm{n}}}}^{L_{\mathrm{s} / \mathrm{c}}} N_{\mathrm{e}}(l) \mathrm{d} L$

$n_{\text {cri }}(f)=1.240 \times 10^{4}\left(\frac{f}{1 \mathrm{MHz}}\right)^{2} \mathrm{~cm}^{-3}$,

where $c$ is the speed of light, $n_{\text {cri }}$ is the critical plasma density for the radio carrier frequency $f$, and $N_{\mathrm{e}}$ is an electron density in the unit of electrons per $\mathrm{cm}^{3}$ and is expressed as (Bird et al. 1996)

$N_{\mathrm{e}}(l, \theta)=B\left(\frac{l}{R_{\odot}}\right)^{-\epsilon} F(\theta) \mathrm{cm}^{-3}$,

where $B$ and $\epsilon$ are the real positive parameters to be determined from the data. $R_{\odot}$ and $l$ are the solar radius and radial distance in AU. $F(\theta)$ is the heliolatitude dependency of the electron density (Bird et al. 1996), where $\theta$ represents the heliolatitude location of a point along the LOS at a given epoch. The maximum contribution in the electron density occurs when $l$ equals the minimum distance of the line of sight (MDLOS), $p$ (see Fig. 3), from the Sun. At a given epoch, MDLOS is estimated from the planetary and spacecraft ephemerides. The ratio of the MDLOS and the solar radii $\left(R_{\odot}\right)$ is given by $r$, which is also called the impact factor:

$\left(\frac{p}{R_{\odot}}\right)=r$

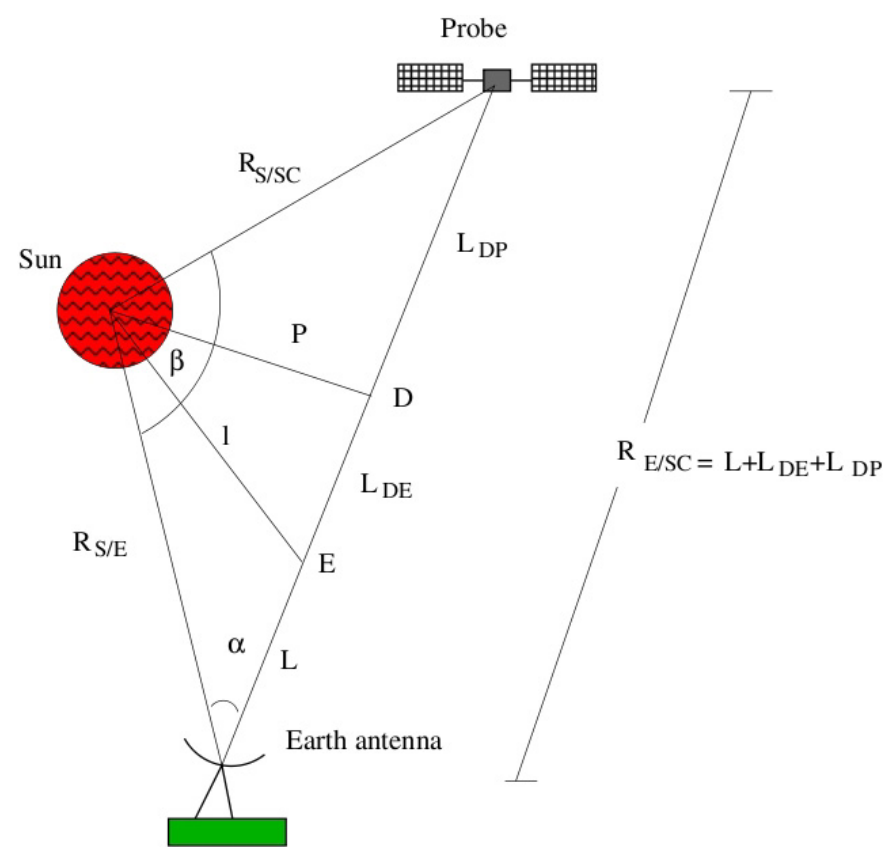

Fig. 3. Geometric relation between Earth-Sun-Probe. Where $\beta$ is the Earth-Sun-Probe (ESP) angle and $\alpha$ is the Sun-Earth-Probe (SEP) angle.

The electron density profile presented by Bird et al. (1996) is valid for MDLOS greater than $4 R_{\odot}$. Below this limit, turbulences and irregularities are very high and non-negligible. The solar plasma is therefore considered as inhomogeneous and additional terms (such $r^{-6}$ and $r^{-16}$ ) could be added to Eq. (2) 
(Muhleman et al. 1977; Bird et al. 1994). However, because of the very high uncertainties in the spacecraft orbit and range bias measurements within these inner regions, we did not include these terms in our solar corona corrections.

At a given epoch, the MGS, MEX, and VEX MDLOS always remain in the ecliptic plane. The latitudinal variations in the coverage of data are hence negligible compared to the variation in the MDLOS. These data sets are thus less appropriate for the analysis of the electron density as a function of heliolatitude (Bird et al. 1996). Equation (2) can therefore be expressed as a function of the single-power-law $(\epsilon)$ of radial distance only and be reduced to

$N_{\mathrm{e}}(l)=B\left(\frac{l}{R_{\odot}}\right)^{-\epsilon} \mathrm{cm}^{-3}$.

On the other hand, at a given interval of the MDLOS in the ecliptic plane, Guhathakurta et al. (1996) and Leblanc et al. (1998) added one or more terms to Eq. (3), that is

$N_{\mathrm{e}}(l)=A\left(\frac{l}{R_{\odot}}\right)^{-c}+B\left(\frac{l}{R_{\odot}}\right)^{-d} \mathrm{~cm}^{-3}$

with $c \simeq 4$ and $d=2$.

To estimate the travel-time delay, we analytically integrated the LOS (Eq. (1)) from the Earth station to the spacecraft, using Eq. (3) and Eq. (4) individually. The analytical solutions of these integrations are given in Appendix A.

In general, the parameters of the electron density profiles differ from one model to another. These parameters may vary with the type of observations, with the solar activity, or with the solar wind state. In contrast, the primary difference between the several models postulated for the electron density $(r>4)$ is the parameter $\epsilon$ (Eq. (3)), which can vary from 2.0 to 3.0.

For example, the density profile parameters obtained by Muhleman et al. (1977) using the Mariner-7 radio science data for the range of the MDLOS from $5 R_{\odot}$ to $100 R_{\odot}$ at the time of maximum solar cycle phase are

$N_{\mathrm{e}}=\frac{(1.3 \pm 0.9) \times 10^{8}}{r^{6}}+\frac{(0.66 \pm 0.53) \times 10^{6}}{r^{2.08 \pm 0.23}} \mathrm{~cm}^{-3}$.

The electron density profile derived by Leblanc et al. (1998) using the data obtained by the Wind radio and plasma wave investigation instrument, for the range of the MDLOS from about $1.3 R_{\odot}$ to $215 R_{\odot}$ at the solar cycle minimum is

$N_{\mathrm{e}}=\frac{0.8 \times 10^{8}}{r^{6}}+\frac{0.41 \times 10^{7}}{r^{4}}+\frac{0.33 \times 10^{6}}{r^{2}} \mathrm{~cm}^{-3}$.

Furthermore, based on in situ measurements, such as those obtained with the Helios 1 and 2 spacecraft, Bougeret et al. (1984) gave an electronic profile as a function of the MDLOS from $64.5 R_{\odot}$ to $215 R_{\odot}$ as follows

$N_{\mathrm{e}}=\frac{6.14}{p^{2.10}} \mathrm{~cm}^{-3}$.

Similarly, Issautier et al. (1998) analyzed in situ measurements of the solar wind electron density as a function of heliolatitude during a solar minimum. The deduced electron density profile at high latitude $\left(>40^{\circ}\right)$ is given as

$N_{\mathrm{e}}=\frac{2.65}{p^{2.003 \pm 0.015}} \mathrm{~cm}^{-3}$.

This Ulysses high-latitude data set is a representative sample of the stationary high-speed wind. This offered the opportunity to study the in situ solar wind structure during the minimal variations in the solar activity. As presented in Issautier et al. (1998), electronic profiles deduced from other observations in numerous studies were obtained either during different phases of solar activity (minimum or maximum), different solar wind states (fast or slow-wind), using data in the ecliptic plane (low latitudes). These conditions may introduce some bias in the estimation of electronic profiles of density.

Comparisons of the described profiles with the results obtained in this study are presented in Sect. 4.2 and are plotted in Fig. 9.

\subsection{Solar wind identification of LOS and data fitting}

As described in Schwenn (2006), the electronic profiles are very different in slow- and fast-wind regions. In slow-wind regions, one expects a higher electronic density close to the MNL of the solar corona magnetic field at low latitudes (You et al. 2007). It is then necessary to identify if the region of the LOS is either affected by the slow-wind or by the fast-wind. To investigate that question, we computed the projection of the MDLOS on the Sun surface. We then located the MDLOS heliographic longitudes and latitudes with the maps of the solar corona magnetic field as provided by the $\mathrm{WSO}^{3}$. This magnetic field is calculated from photospheric field observations with a potential field model ${ }^{4}$.

However, zones of slow-wind are variable and not precisely determined (Mancuso \& Spangler 2000; Tokumaru et al. 2010). As proposed in You et al. $(2007,2012)$, we took limits of the slow solar wind regions as a belt of $20^{\circ}$ above and below the MNL during the solar minima. For the 2002 solar maximum, this hypothesis is not valid, the slow-wind region being wider than during solar minima. Tokumaru et al. (2010) showed the dominating role of the slow-wind for this entire period and for latitudes lower than $\pm 70^{\circ}$ degrees.

An example of the MDLOS projection on the Sun's surface with the maps of solar corona magnetic field is shown in Fig. 4. These magnetic field maps correspond to the mean epoch of the ingress and egress phases of the solar conjunction at $20 R_{\odot}$. The dark solid line represents the MNL and the belt of the slowwind region is presented by dashed lines. The two marked points give the projected locations of the MDLOS, ingress $(\boldsymbol{\Delta})$ and egress $(\boldsymbol{\nabla})$ at $20 R_{\odot}$. For the entire MDLOS, this distribution of the slow- or fast-wind regions in the ingress and egress phases of solar conjunctions are shown in Fig. 5. The black (white) bars in Fig. 5 present the count of data sets distributed in the slow(fast-) wind region.

After separating the MDLOS into slow- and fast-wind regions as defined in Fig. 5, the parameters of Eqs. (3) and (4) are then calculated using least-squares techniques. These parameters are obtained for various ranges of the MDLOS, from $12 R_{\odot}$ to $215 R_{\odot}$ for MGS, $6 R_{\odot}$ to $152 R_{\odot}$ for MEX, and from $12 R_{\odot}$ to $154 R_{\odot}$ for VEX. The adjustments were performed, for all available data acquired at the time of the solar conjunctions, for each spacecraft individually, and separately for fast- and slowwind regions (see Table 2).

To estimate the robustness of the electronic profile determinations, adjustments on ingress and egress phases were performed separately. The differences between these two estimations and the one obtained on the whole data set give the sensitivity of the profile fit to the distribution of the data, but also to the solar wind states. These differences are thus taken as

\footnotetext{
http://wso.stanford.edu/

4 http://wso.stanford.edu/synsourcel.html
} 

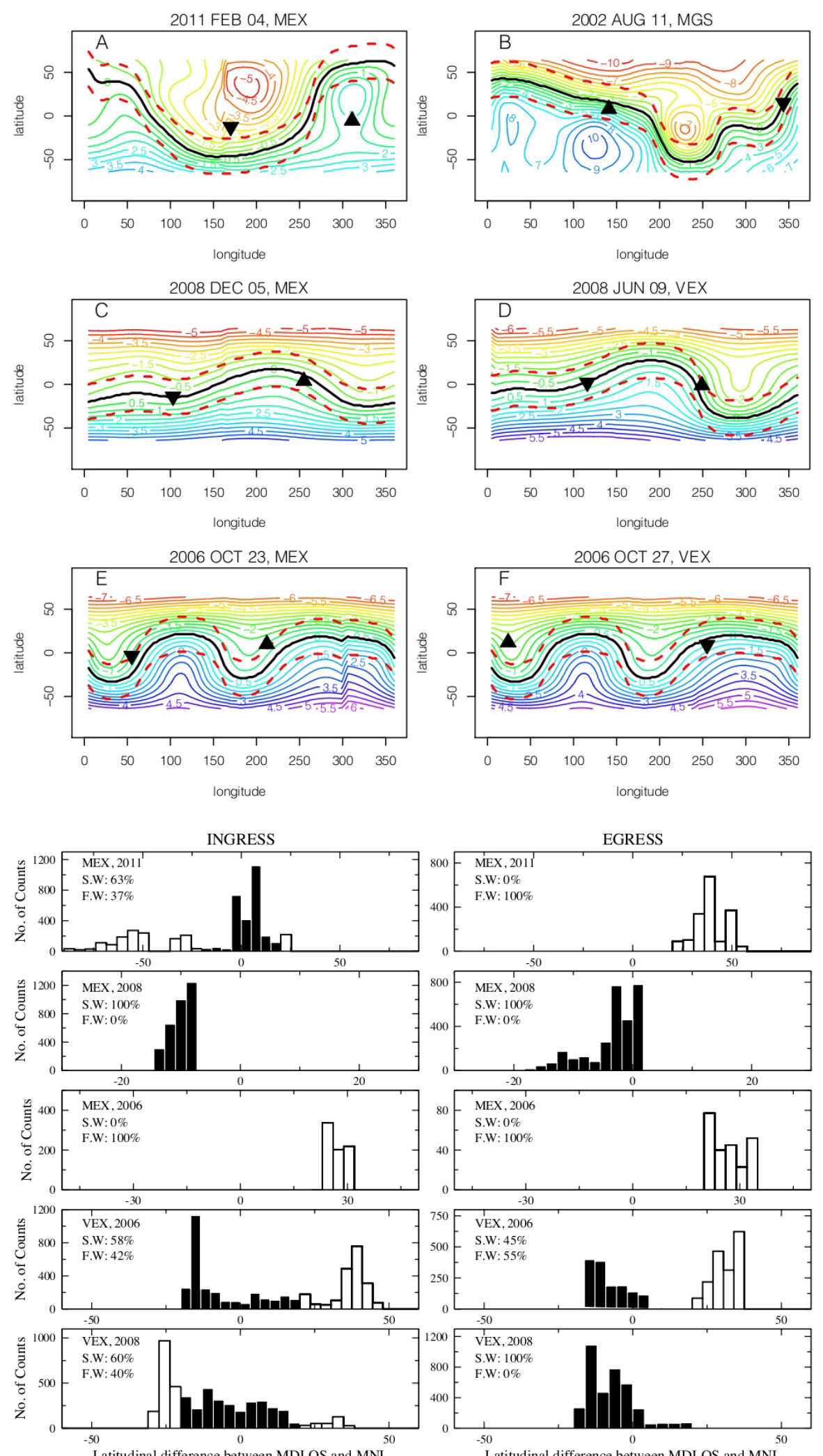

Fig. 4. Solar corona magnetic field maps extracted from Wilcox Solar Observatory (WSO) at the mean epoch of $20 R_{\odot}$ ingress and egress. The dark solid line represents the magnetic neutral line. The dashed red lines correspond to the belt of the slow-wind region. The two marked points give the projected locations of the ingress $(\mathbf{\Delta})$ and egress ( $\mathbf{v})$ minimal distances at $20 R_{\odot}$. For the MGS 2002 solar conjunction, the hypothesis of a $\pm 20^{\circ}$ belt is not relevant (see Sect. 4.1.1).

Fig. 5. Distribution of the latitudinal differences between MDLOS and magnetic neutral line (MNL) in the slow- and fast-wind regions during the ingress and egress phases of solar conjunctions. The black (white) bars present the slow(fast-) wind regions as defined by $\pm 20^{\circ}\left(> \pm 20^{\circ}\right)$ along the MNL during solar minima.

the uncertainty in the estimations and are given as error bars in the Table 2.

\section{Results and discussions}

\subsection{Estimated model parameters and electron density}

As described in Sect. 3.2, we estimated the model parameters and the electron density separately for each conjunction of the
MGS, MEX, and VEX. A summary of these results is presented in Table 2. The MDLOS in the unit of solar radii $\left(R_{\odot}\right)$ mentioned in this table (Col. 5) represents the interval of available data used for calculating the electronic profiles of density. These profiles were then used for extrapolating the average electron density at $215 R_{\odot}(1 \mathrm{AU})$. The period of the solar conjunctions, solar activities, and solar wind states are also given in Cols. 2-4. Table 2 also contains the estimated parameters of 


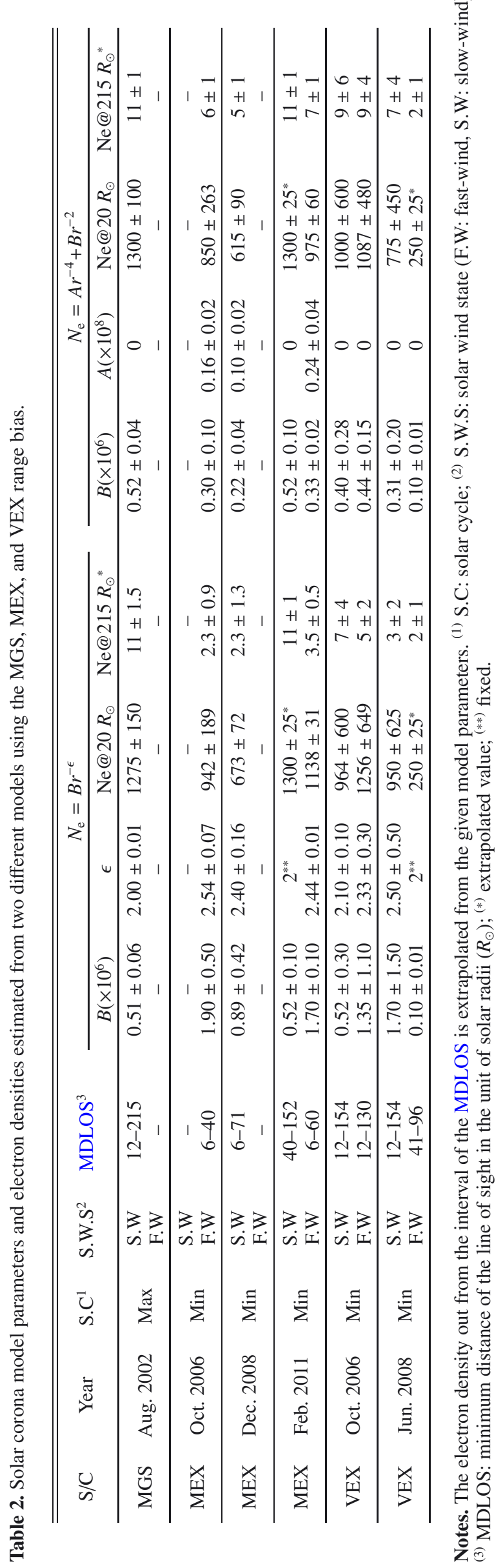

two different models: the first model from Bird et al. (1996) corresponds to Eq. (3), whereas the second model is based on Guhathakurta et al. (1996) and Leblanc et al. (1998) and follows Eq. (4) with $c=4$. Estimated model parameters for the slowand fast-wind regions are presented in Cols. 6 and 7 .

\subsubsection{Mars superior conjunction}

The MGS experienced its superior conjunction in 2002 when the solar activity was maximum and the slow-wind region was spread at about $\pm 70^{\circ}$ of heliolatitude (Tokumaru et al. 2010). Figure 6 represents the projection of the MGS MDLOS on the solar surface (black dots) superimposed with the 2002 synoptic source surface map of solar wind speeds derived from STEL IPS observations extracted from Tokumaru et al. (2010). It suggests that, the MDLOS of the MGS exclusively remains in the slow-wind region. Respective estimates of the model parameters and of the electron densities are given in Table 2. From this table one can see that the estimates of the electron density from both models are very similar. Parameter $\epsilon$ of Eq. (3) is then estimated as $2.00 \pm 0.01$, which represents a radially symmetrical behavior of the solar wind and hence validates the assumption of a spherically symmetrical behavior of the slow-wind during solar maxima (Guhathakurta et al. 1996). Whereas for Eq. (4), the contribution of the $r^{-4}$ term at large heliocentric distances $\left(r>12 R_{\odot}\right)$ is negligible compared to the $r^{-2}$ term. Thus, the parameter $A$ for these large heliocentric distances is fixed to zero and consequently gave similar results to Eq. (3), as shown in Table 2.

In contrast, MEX experienced its superior conjunctions in 2006, 2008, and 2011 during solar minima. The distribution of data during these conjunctions with respect to MDLOS are shown in Fig. 7. From this figure, one can see that the MEX 2006 (2008) conjunction corresponds to the fast- (slow-) wind region, whereas 2011 conjunction is a mixture of slow- and fastwinds. The estimated parameters of these conjunctions are given in Table 2. An example of the comparison between two models (Eqs. (3) and (4)) is shown in Fig. 8. This figure compares the electron density profiles obtained from the two models during the MEX 2008 conjunction. These profiles are extrapolated from $1 R_{\odot}$ to $6 R_{\odot}$ and from $71 R_{\odot}$ to $215 R_{\odot}$. The upper triangles in Fig. 8 indicate electronic densities obtained at $20 R_{\odot}$ and at the extrapolated distance of $215 R_{\odot}(1 \mathrm{AU})$ with error bars obtained as described in Sect. 3.2. As one sees in that figure, electronic profiles are quite similar over the computation interval till $71 R_{\odot}$ and become significantly different after this limit. This suggests that the estimated parameters for both models are valid for the range of MDLOS given in Table 2. Finally, for the MEX 2011 conjunction, as shown in Figs. 5 and 7, the data are mainly distributed in the slow-wind $(63 \%)$ during ingress phase and in the fast-wind (100\%) during egress phase. Owing to the unavailability of slow-wind data near the Sun (MDLOS $<40 R_{\odot}$ ), we fixed $\epsilon$ to 2 for Eq. (3) (see Table 2). Moreover, the average electron density estimated for this conjunction is higher for the slow-wind than for the fast-wind and it is consistent with Tokumaru et al. (2010), which suggests that near the MNL, the electron content is higher than in the fast-wind regions.

\subsubsection{Venus superior conjunction}

The VEX 2006 and 2008 conjunctions exhibit a mixture of slow- and fast-wind (Fig. 7). These conjunctions occurred approximately at the same time as the MEX superior conjunctions. 


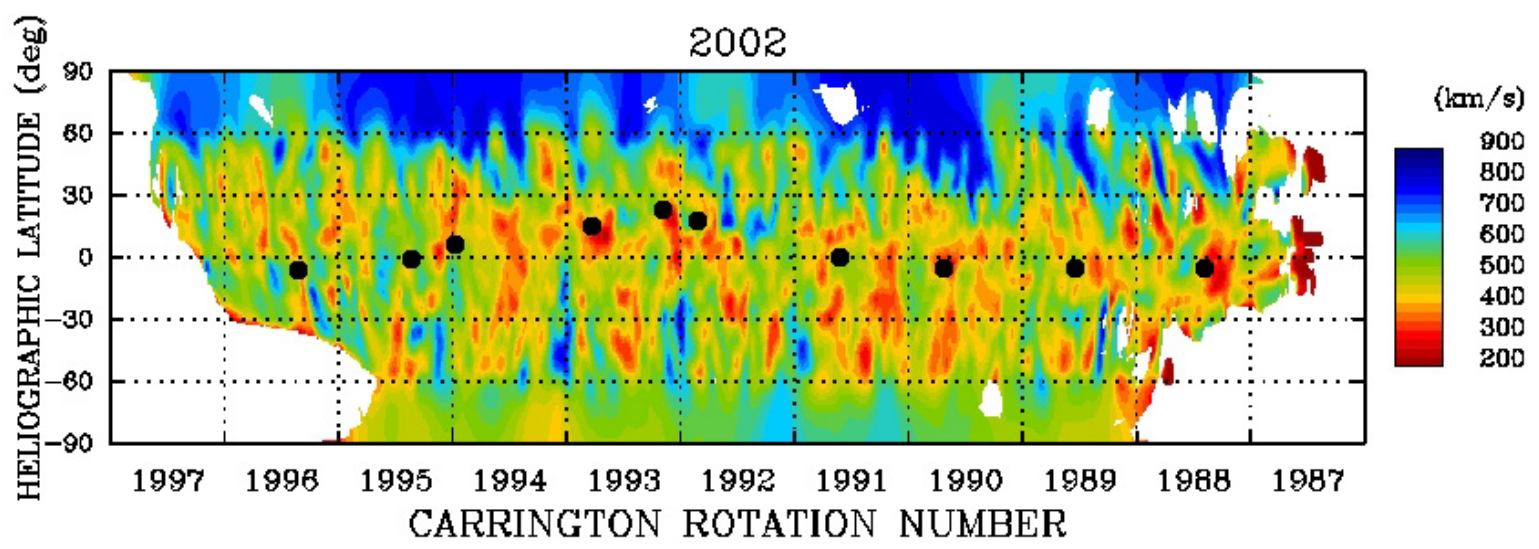

Fig. 6. 2002 synoptic source surface map of solar wind speeds derived from STEL IPS observations extracted from Tokumaru et al. (2010). The black dots represent the MGS MDLOS during the 2002 conjunction period for the range of $12 R_{\odot}$ to $120 R_{\odot}$.
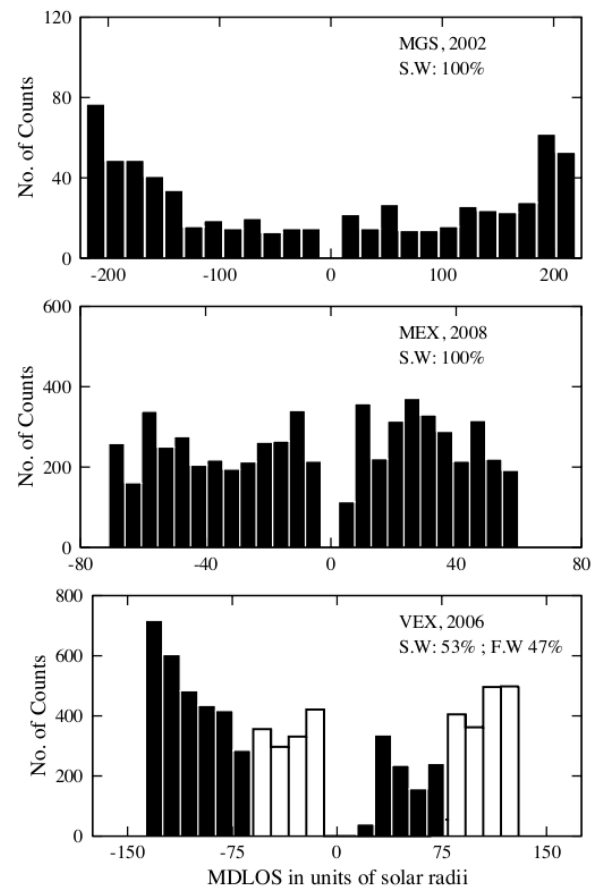
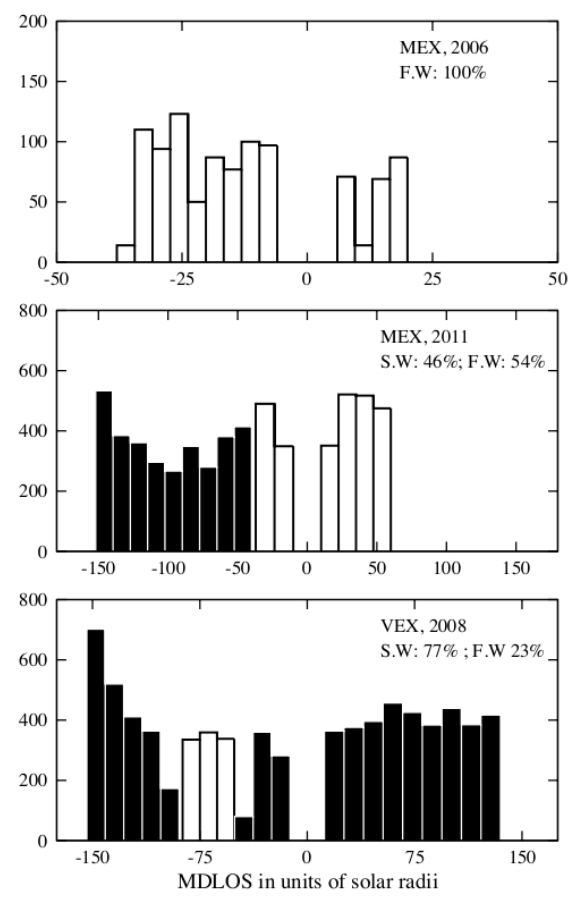

Fig. 7. Distribution of the MGS, MEX, and VEX data in the slow (black) and fast (white) wind with respect to MDLOS in units of solar radii $\left(R_{\odot}\right)$. Negative (positive) MDLOS represents the distribution in the ingress (egress) phase.

Table 3. Electron densities estimated from different models at $20 R_{\odot}$ and at $215 R_{\odot}(1 \mathrm{AU})$.

\begin{tabular}{|c|c|c|c|c|c|}
\hline Authors & Spacecraft & $\begin{array}{c}\text { Solar } \\
\text { activity }\end{array}$ & MDLOS & $\begin{array}{c}N e @ 20 R_{\odot} \\
\left(\mathrm{el} . \mathrm{cm}^{-3}\right)\end{array}$ & $\begin{array}{c}N e @ 215 R_{\odot} \\
\left(\text { el. cm }{ }^{-3}\right)\end{array}$ \\
\hline Leblanc et al. (1998) & Wind & Min & $1.3-215$ & 847 & 7.2 \\
\hline Bougeret et al. (1984) & Helios 1 and 2 & $\operatorname{Min} / \operatorname{Max}$ & $65-215$ & 890 & 6.14 \\
\hline Issautier et al. (1998) & Ulysses & Min & $327-497$ & $307^{*}$ & $2.65 \pm 0.5^{*}$ \\
\hline Muhleman et al. (1977) & Mariner 6 and 7 & Max. & $5-100$ & $1231 \pm 64$ & $9 \pm 3$ \\
\hline Bird et al. (1994) & Ulysses & $\operatorname{Max}$ & $5-42$ & $1700 \pm 100$ & $4.7 \pm 0.415$ \\
\hline Anderson et al. (1987) & Voyager 2 & Max & $10-88$ & $6650 \pm 850$ & $38 \pm 4$ \\
\hline
\end{tabular}

Notes. ${ }^{(*)}$ Mean electron density corresponds to latitude $\geq 40^{\circ}$.

However, the limitations in the VEX orbit determination introduced bias in the estimation of the model parameters and the electron densities. This can be verified from the discrepancies presented in Table 2 for the VEX 2006 and 2008 conjunctions. Despite these high uncertainties, post-fit range bias corrected for the solar corona allows one to add complementary data in the construction of the planetary ephemerides (see Sect. 4.5).

\subsection{Comparison with other models}

Table 3 represents the estimated electron densities at $20 R_{\odot}$ and $215 R_{\odot}(1 \mathrm{AU})$ from the various models. These models are representative of radio science measurements (Muhleman et al. 1977; Anderson et al. 1987; Bird et al. 1994, 1996), in situ measurements (Bougeret et al. 1984; Issautier et al. 1997), and 


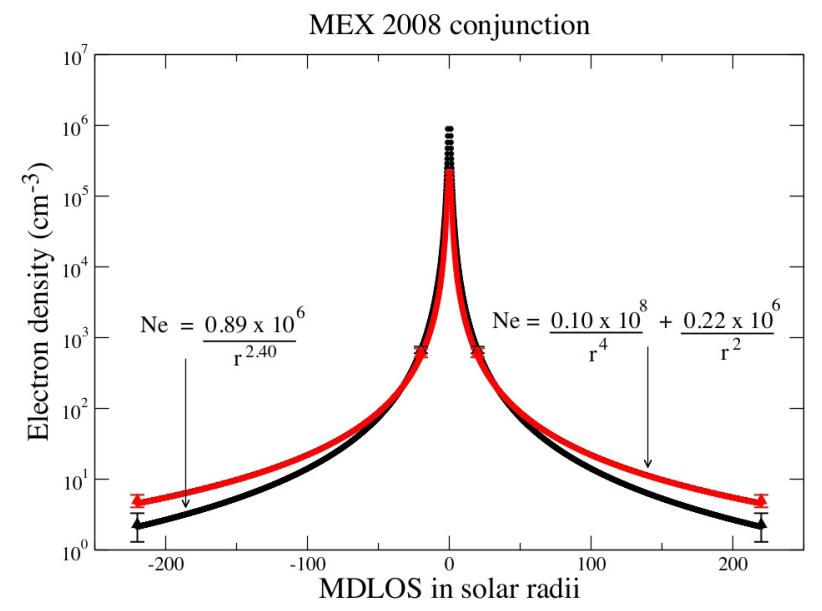

Fig. 8. Example of the comparison between electron density models given in Eqs. (3) and (4). The electron density profiles are plotted from $1 R_{\odot}$ to $215 R_{\odot}(1 \mathrm{AU})$ for the MEX 2008 conjunction using the model parameters given in Table 2 . The error bars plotted in the figure correspond to the electron density obtained at $20 R_{\odot}$ and $215 R_{\odot}$ (see Table 2).

solar type III radio emission (Leblanc et al. 1998) measurements (Table 1). Table 3 and Fig. 9 allow us to compare the average electron density, obtained from different observations, made approximately during the same solar activity cycle.

Figure 9 illustrates the comparisons of different electron density profiles, extrapolated from $1 R_{\odot}$ to $215 R_{\odot}$. From this figure it can be seen that approximately all electron density profiles follow similar trends ( $\propto r^{-\epsilon}, \epsilon$ varying from 2 to 3 ) until $10 R_{\odot}$ (panel B), whereas the dispersions in the profiles below $10 R_{\odot}$ (panel A) are due to the contribution of higher order terms, such as $r^{-4}, r^{-6}$ or $r^{-16}$.

In Table 3, we also provide the average electron density at $20 R_{\odot}$ and $215 R_{\odot}$, based on the corresponding models (if not given by the authors). The two individual electron density profiles for ingress and egress phases have been given by Anderson et al. (1987) and Bird et al. (1994). To compare their estimates with ours, we took the mean values of both phases. Similarly, Muhleman et al. (1977) gave the mean electron density at $215 R_{\odot}$ estimated from round-trip propagation time delays of the Mariner 6 and 7 spacecraft.

Table 3 shows a wide range of the average electron densities, estimated at $20 R_{\odot}$ and $215 R_{\odot}$ during different phases of solar activity. Our estimates of the average electron density shown in Table 2 are very close to the previous estimates, especially during solar minimum. The widest variations between our results and the earlier estimates were found during solar maxima and can be explained from the high variability of the solar corona during these periods.

\subsection{Post-fit residuals}

One of the objectives of this study is to minimize the effect of the solar corona on the range bias. These post-fit range biases can then be used to improves the planetary ephemeris (INPOP). The pre-fit range bias represents the systematic error in the planetary ephemerides during the solar conjunction periods. Figure 10 shows the pre-fit residuals over plotted with the simulated time delay (in units of distance), obtained from the solar corona model based on Eq. (3). In contrast, the post-fit range bias represents the error in the ephemerides after correction for solar corona perturbations.
Table 4. Statistics of the range bias before and after solar corona corrections.

\begin{tabular}{lccccc}
\hline \hline \multirow{2}{*}{ S/C } & \multicolumn{2}{c}{ Pre-fit } & & \multicolumn{2}{c}{ Post-fit } \\
\cline { 2 - 3 } \cline { 5 - 6 } & mean $(\mathrm{m})$ & $\sigma(\mathrm{m})$ & & mean $(\mathrm{m})$ & $\sigma(\mathrm{m})$ \\
\hline MGS, 2002 & 6.02 & 10.10 & & -0.16 & 2.89 \\
MEX, 2006 & 42.03 & 39.30 & & 0.85 & 9.06 \\
MEX, 2008 & 16.00 & 20.35 & & -0.10 & 4.28 \\
MEX, 2011 & 15.44 & 19.20 & & 0.11 & 6.48 \\
VEX, 2006 & 5.47 & 11.48 & & -0.74 & 6.72 \\
VEX, 2008 & 3.48 & 11.48 & & -0.87 & 7.97 \\
\hline
\end{tabular}

From Fig. 10 one can see that the systematic trend of the solar corona perturbations is almost removed from the range bias. The post-fit range bias of the VEX at low solar radii (especially during the egress phase) is not as good as the MGS and MEX. This can be explained by the degraded quality of the VEX orbit determination (see Sect. 2.2.2). The dispersion in the pre-fit and post-fit range bias is given in Table 4 . The estimated dispersions in the post-fit range bias are one order of magnitude lower than the dispersions in the pre-fit range bias. It shows a good agreement between the model estimates and the radiometric data. The corrected range bias (post-fit) is then used to improve the planetary ephemerides (see Sect. 4.5).

\subsection{Model parameter dependency on the ephemerides}

The range bias data are usually very important for constructing the planetary ephemerides (Folkner et al. 2008; Fienga et al. 2011b). These measurements correspond to at least $57 \%$ of the total amount of data used for the INPOP construction (Fienga et al. 2009). Range bias data at the time of the solar conjunctions are not taken into account due to very high uncertainties (see Fig. 2). Equation (3) and Fig. 3 show the dependency of the density profile over geometric positions of the spacecraft (orbiting a planet) relative to the Earth and the Sun. The range bias used for this study includes the error in the geometric distance of Mars and Venus relative to the Earth. These errors are varying from one ephemeris to another and impact directly on the estimates of the mean electron density.

Figure 11 illustrates the electron density estimated at $20 R_{\odot}$ for MGS, MEX and VEX using DE421 and INPOP10b ephemerides. The dashed (DE421) and dotteddashed (INPOP10b) lines present the time limit up to which these ephemerides are fitted over range bias data. The curve represents the differences between INPOP10b and DE421 estimations of Mars-Earth geometric distances.

In particular, the Mars orbit is affected by the belt of asteroids. The asteroid masses may cause a degradation in the estimates of the Mars orbit. Therefore, as one can see in Fig. 11, the geometric differences between the INPOP10b and DE421 estimates of the Mars-Earth geometric distances are magnified from the extrapolation period onward. Hence, the electron densities estimated using DE421 and INPOP10b are consistent with each other within the error bars before the extrapolation period, whereas after this period, the DE421and INPOP10b estimates of the electron densities are quite different from each other. The sensitivity of the solar corona parameters and the electron densities, deduced from the analysis of the range bias, is then low as long as the computation is included in the time interval of the fit of the planetary ephemerides. However, out from the fitting time, the quality of these computations can be degraded by the extrapolation capability of the planetary ephemerides. Conversely, by 

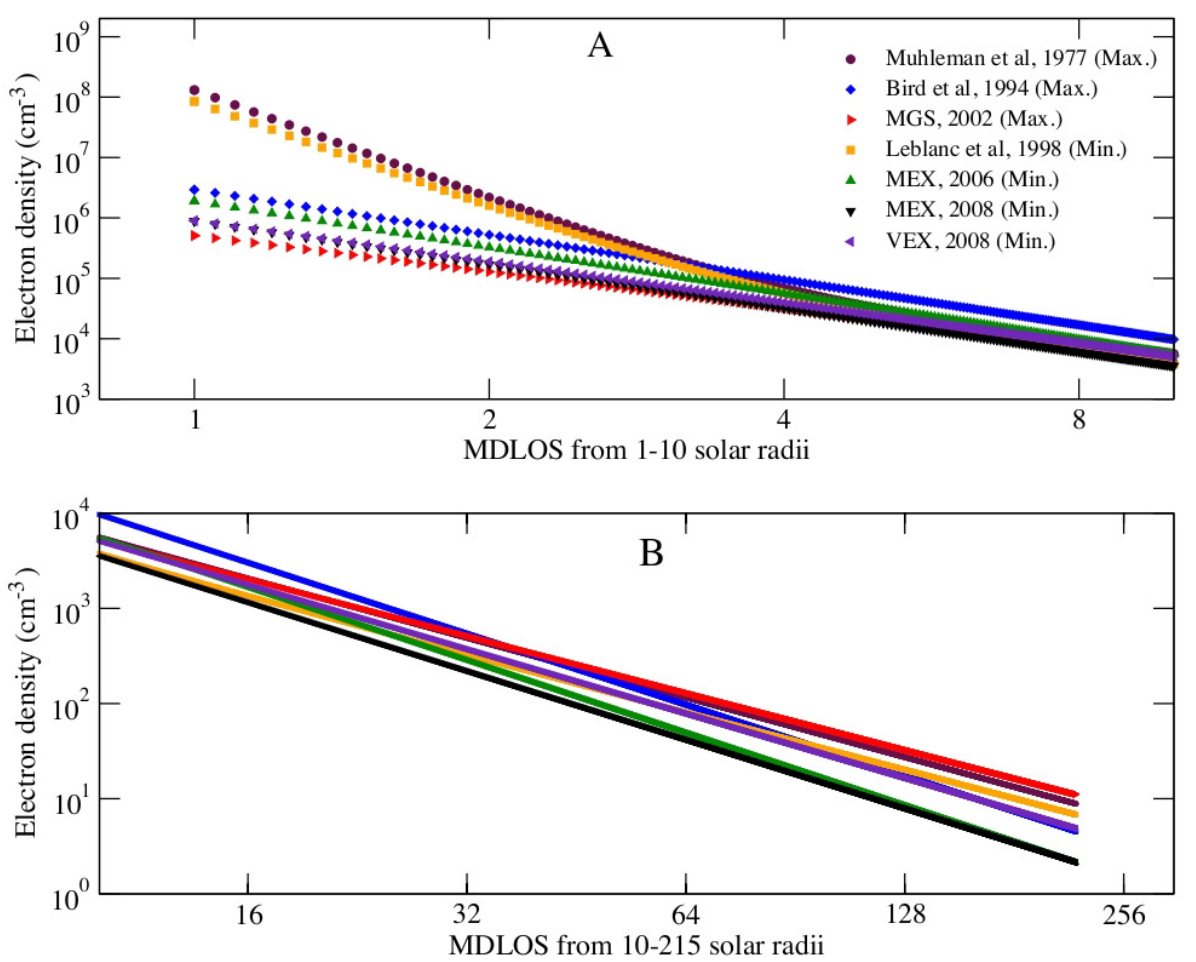

Fig. 9. Comparison of different electron density profiles at different phases of the solar cycle from $1 R_{\odot}$ to $215 R_{\odot}(1 \mathrm{AU})$. fitting the planetary ephemerides (INPOP) including data corrected for the solar corona perturbations, some noticeable improvement can appear in the extrapolation capability of the planetary ephemerides and in the estimates of the asteroid masses (see Sect. 4.5).

\subsection{Impact on planetary ephemerides}

As one can see in Fig. 10, correct the effects induced by the solar corona on the observed Mars-Earth distances is significant over some specific periods of time (during solar conjunctions). We aim to estimate the impact of this important but time-limited improvement of the measurements of interplanetary distances on the construction of the planetary ephemerides. To evaluate any possible improvement, we produced two ephemerides, INPOP10c and INPOP10d, both fitted over the same data set as was used for the construction of INPOP10b (Fienga et al. 2011b). This data set contains all planetary observations commonly used for INPOP (see Fienga et al. 2009, 2011a), including the MGS data obtained in section 2.1.1 and the MEX and VEX range bias provided by ESA. These newly built ephemerides are based on the same dynamical modeling as described in Fienga et al. (2009, 2011a). However, INPOP10c is estimated without any solar corona corrections on the MGS, MEX and VEX range bias, and INPOP10d includes the solar corona corrections evaluated in the previous sections. The selection of the fitted asteroid masses and the adjustment method (bounded value least-squares associated with a priori sigmas) are the same for the two cases. The weighting schema are also identical. The differences remain in the quality and the quantity of the range bias used for the fit (one corrected for solar plasma and one not) and in the procedure selecting the data actually used in the fit.

For INPOP10c, about 119901 observations were selected. Of these, $57 \%$ are MGS, MEX, and VEX range bias data that are not corrected for solar corona effects. Based on a very conservative procedure, observations obtained two months before and after the conjunctions were removed from the fitted data sample. This strategy leads to removal of about $7 \%$ of the whole data set, which represents $14 \%$ of the MGS, MEX, and VEX observations. For INPOP10d, thanks to the solar corona corrections, only observations of SEP smaller than 1.8 degrees were removed from the data sample. This represents less than $1 \%$ of the whole data sample. The estimated accuracy of the measurements corrected for the solar plasma is 2.4 meters when observations not affected by the solar conjunctions have an accuracy of about $1.7 \mathrm{~m}$. By keeping more observations during solar conjunction intervals, the number of data with a good accuracy is then significantly increased.

Adjustments of planet initial conditions, mass of the sun, sun oblateness, mass of an asteroid ring, and the masses of 289 asteroids were then performed in the same fitting conditions as INPOP10b.

No significant differences were noted for the evaluated parameters except for the asteroid masses.

For the masses estimated both in INPOP10c and INPOP10d, $20 \%$ induce perturbations bigger than $5 \mathrm{~m}$ on the Earth-Mars distances during the observation period. The masses of these $59 \mathrm{ob}-$ jects are presented in Table 5. Within the 1- $\sigma$ uncertainties deduced from the fit, we notice $10(17 \%)$ significant differences in masses obtained with INPOP10c and INPOP10d, quoted with a "*" in Col. 5 of the Table, $7(12 \%)$ new estimates made with INPOP10d, noted $\mathrm{N}$ in Cols. 5, and $6(10 \%)$ masses put to 0 in INPOP10d when estimated in INPOP10c, marked with 0 in the fifth column.

Table 6 lists masses found in the literature compared with the quoted values of Table 5 . In this Table, $80 \%$ of the INPOP10d estimates have a better consistency with the values obtained by close encounters than the one obtained with INPOP10c. Of these, the new estimates obtained with INPOP10d for (20), (139) and (27) agree well with the values found in the literature. For (45) Eugenia, the INPOP10c value is closer to the mass deduced from the motion of its satellite (Marchis et al. 2008) even if the INPOP10d value is still compatible at 2- $\sigma$. For (130) Elektra, the INPOP10c and INPOP10d estimated values are certainly under evaluated. Finally, one can note the systematic bigger 


\section{A. K. Verma et al.: Electron density distribution from MGS, MEX, and VEX}
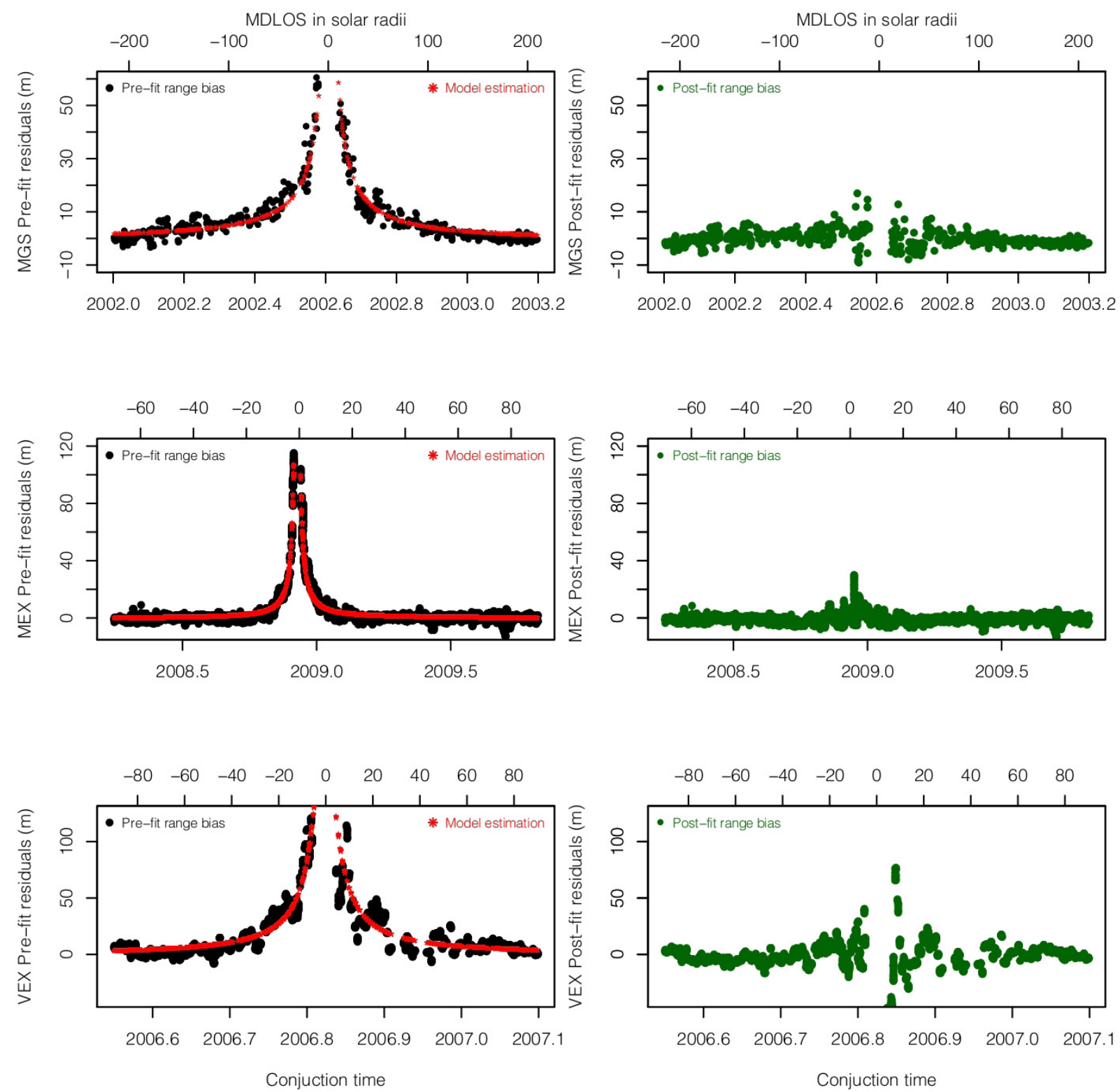

Fig. 10. Left panel: model-estimated solar corona over-plotted on the pre-fit residuals. Right panel: post-fit residuals after corona corrections. Top, middle, and bottom panels correspond to the MGS 2002, MEX 2008, and VEX 2006 conjunctions.

uncertainties of the INPOP10d estimates. The supplementary data sample collected during the solar conjunctions that has $30 \%$ more noise than the data collected beyond the conjunction can explain the degradation of the uncertainties for the INPOP10d determinations compared to INPOP10c.

By correcting the range bias for the solar corona effects, we added more informations related to the perturbations induced by the asteroids during the conjunction intervals.

In principal, during the least-squares estimation of the asteroid masses, the general trend of the gravitational perturbation induced by the asteroid on the planet orbits should be described the most completely by the observable (the Earth-Mars distances) without any lack of information. In particular, for an optimized estimation, the data sets used for the fit should include local maxima of the perturbation.

However, it could happen that some of the local maxima occur during the solar conjunction intervals. One can then expect a degradation of the least-squares estimation of the perturber mass if no solar corrections are applied or if these intervals are not taken into account during the fit. To estimate which mass determination can be more degraded than another by this window effect, we estimated $L$, the percentage of local maxima rejected from the INPOP10c fit in comparison with the INPOP10d adjustment including all data sets corrected for solar plasma. $L$ will give the loss of information induced by the rejection of the solar conjunction intervals in terms of highest perturbations.

The $L$ criteria are given in Col. 7 of Table 5 . As an example, for (24) Themis one notes in Table 6 the good agreement between the close encounter estimates and the INPOP10d mass determination compared with INPOP10c. On the other hand, based on the $L$ criteria, $36 \%$ of the local maxima happen near solar conjunctions. By neglecting the solar conjunction intervals, more than a third of the biggest perturbations are missing in the adjustment. This can explain the more realistic INPOP10d estimates compared with INPOP10c.

We also indicate in Table 5 if important constraints were added in the fit (Col. 8). In these cases, even if new observations are added to the fit (during the solar conjunction periods), 


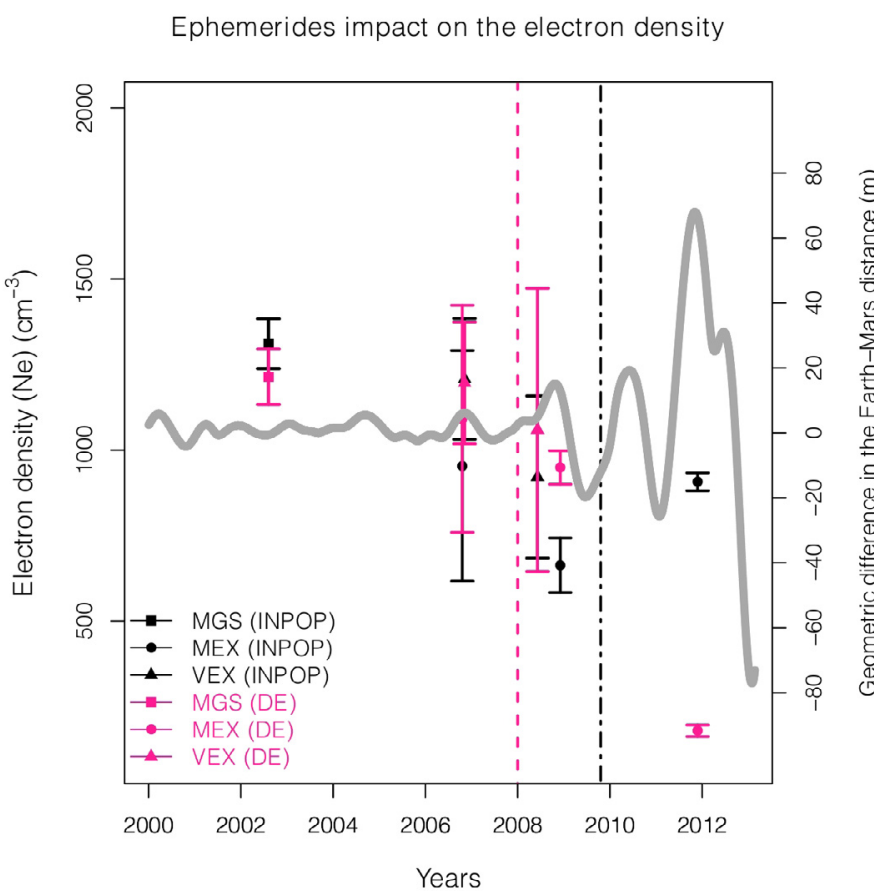

Fig. 11. Variation of the average electron density at $20 R_{\odot}$ and $215 R_{\odot}$ using the DE421 and INPOP10b ephemeris. The dotted-dashed (INPOP) and dashed (DE421) vertical lines present the starting time of extrapolation. The plain line shows the differences in the Mars-Earth geometric distances estimated with INPOP10b and DE421.

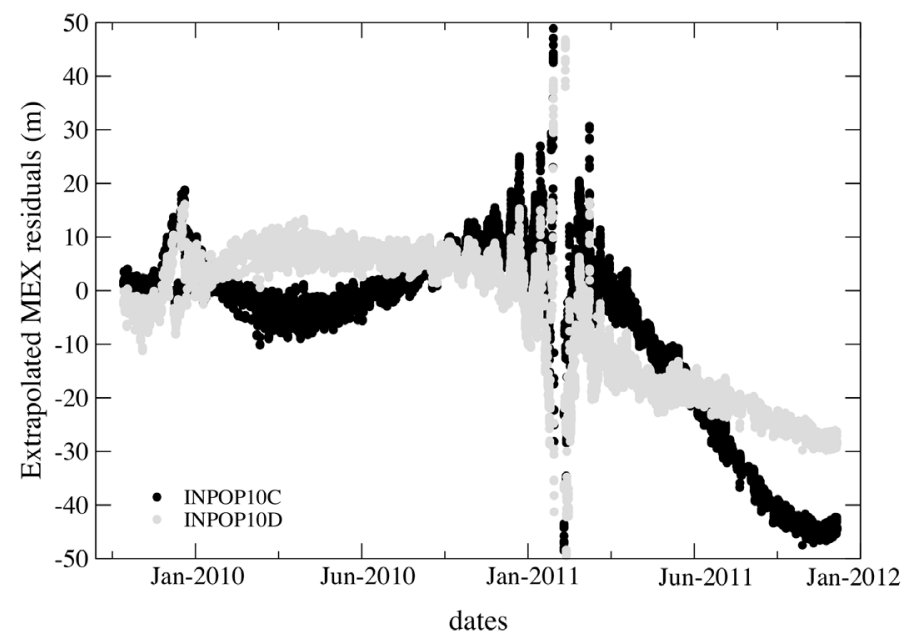

Fig. 12. MEX extrapolated residuals estimated with INPOP10d (light dots) and INPOP10c (dark dots).

there is a high probability to obtain a stable estimates of the constrained masses as for the biggest perturbers of Table 5. For the other mass determinations, one can note a consistency between high values of the $L$ criteria and the non-negligible mass differences between INPOP10c and INPOP10d.

By improving the range bias residuals during the solar conjunction periods, we then slightly improved the asteroid mass determinations.

Estimates of residuals for data samples not used in the INPOP fit and dated after or before the end of the fitting interval are currently made to evaluate the real accuracy of the planetary ephemerides (Fienga et al. 2011a, 2009). To estimate if the use of the solar corona corrections induces a global improvement of the planetary ephemerides, the MEX extrapolated residuals were computed with INPOP10c and INPOP10d. As one can see in Fig. 12, the INPOP10d MEX extrapolated residuals show a better long-term behavior compared with INPOP10c with $30 \%$ less degraded residuals after two years of extrapolation.

Supplementary data of the MEX and VEX obtained during the first six months of 2012 would confirm the long-term evolutions of the INPOP10d, INPOP10c and INPOP10b.

This improvement can be explained by the more realistic adjustment of the ephemerides with denser data sets (7\%) and more consistent asteroid mass fitting.

\section{Conclusion}

We analyzed the navigation data of the MGS, MEX, and VEX spacecraft acquired during solar conjunction periods. We estimated new characteristics of solar corona models and electron densities at different phases of solar activity (maximum and minimum) and at different solar wind states (slow and fast). Good agreement was found between the solar corona model estimates and the radiometric data. We compared our estimates of electron densities with earlier results obtained with different methods. These estimates were found to be consistent during the same solar activities. During solar minima, the electron densities obtained by in situ measurements and solar radio burst III are within the error bars of the MEX and VEX estimates. However, during the solar maxima, electron densities obtained with different methods or different spacecraft show weaker consistencies. These discrepancies need to be investigated in more detail, which requires a deeper analysis of data acquired at the time of solar maxima.

The MGS, MEX, and VEX solar conjunctions data allow us to analyze the large-scale structure of the corona electron density. These analyses provide individual electron density profiles for slow- and fast-wind regions during solar maxima and minima activities.

In the future, planetary missions such as MESSENGER will also provide an opportunity to analyze the radio-science data, especially at the time of maximum solar cycle.

We tested the variability caused by the planetary ephemerides on the electron density parameters deduced from the analysis of the range bias. This variability is smaller than the 1- $\sigma$ uncertainties of the time-fitting interval of the planetary ephemerides but becomes wider beyond this interval. Furthermore, data corrected for solar corona perturbations were used for constructing the INPOP ephemerides. Thanks to these supplementary data, an improvement in the estimation of the asteroid masses and a better behavior of the ephemerides were achieved.

Acknowledgements. A.K. Verma is the research fellow of CNES and Region Franche-Comté and thanks CNES and Region Franche-Comté for financial support. Part of this work was made using the GINS software; we would like to acknowledge CNES, who provided us access to this software. We are also grateful to J.C. Marty (CNES) and P. Rosenbatt (Royal Observatory of Belgium) for their support in handling the GINS software. The Authors are grateful to the anonymous referee for helpful comments, which improved the manuscript.

\section{References}

Anderson, J. D., Krisher, T. P., Borutzki, S. E., et al. 1987, ApJ, 323, L141 Baer, J., Chesley, S. R., \& Matson, R. 2011, AJ, submitted

Bird, M. K., Volland, H., Paetzold, M., et al. 1994, ApJ, 426, 373

Bird, M. K., Paetzold, M., Edenhofer, P., Asmar, S. W., \& McElrath, T. P. 1996, A\&A, 316, 441 
A. K. Verma et al.: Electron density distribution from MGS, MEX, and VEX

Bougeret, J.-L., King, J. H., \& Schwenn, R. 1984, Sol. Phys., 90, 401

Cranmer, S. R. 2002, Space Sci. Rev., 101, 229

Fienga, A., Laskar, J., Morley, T., et al. 2009, A\&A, 507, 1675

Fienga, A., Laskar, J., Manche, H., et al. 2011a, Celestial Mechanics and Dynamical Astronomy

Fienga, A., Kuchynka, P., Laskar, J., et al. 2011b, in EPSC-DPS Join Meeting 2011, 1879

Folkner, W. M., Williams, J. G., \& Boggs, D. H. 2008, IOM 343R-08-003

Guhathakurta, M., \& Holzer, T. E. 1994, ApJ, 426, 782

Guhathakurta, M., Holzer, T. E., \& MacQueen, R. M. 1996, ApJ, 458, 817

Guhathakurta, M., Fludra, A., Gibson, S. E., Biesecker, D., \& Fisher, R. 1999, J. Geophys. Res., 104, 9801

Issautier, K., Meyer-Vernet, N., Moncuquet, M., \& Hoang, S. 1997, Sol. Phys., 172,335

Issautier, K., Meyer-Vernet, N., Moncuquet, M., \& Hoang, S. 1998, J. Geophys. Res., 103, 1969

Kuchynka, P., Laskar, J., Fienga, A., \& Manche, H. 2010, A\&A, 514, A96

Leblanc, Y., Dulk, G. A., \& Bougeret, J.-L. 1998, Sol. Phys., 183, 165

Mancuso, S., \& Spangler, S. R. 2000, ApJ, 539, 480

Marchis, F., Descamps, P., Baek, M., et al. 2008, Icarus, 196, 97
Marty, J. C., Balmino, G., Duron, J., et al. 2009, Planet. Space Sci., 57, 350

Moyer, T. D. 2003, Formulation for Observed and Computed Values of Deep Space Network Data Types for Navigation (John Wiley \& Sons), 2

Muhleman, D. O., \& Anderson, J. D. 1981, ApJ, 247, 1093

Muhleman, D. O., Esposito, P. B., \& Anderson, J. D. 1977, ApJ, 211, 943

Schwenn, R. 2006, Space Sci. Rev., 124, 51

Schwenn, R., \& Marsch, E. 1990, Physics of the Inner Heliosphere, I Large-Scale Phenomena (Springer), Phys. Chem. Space, 20

Schwenn, R., \& Marsch, E. 1991, Physics of the Inner Heliosphere, II Particles, Waves and Turbulence (Springer), Phys. Chem. Space, 21

Standish, E. M. 1998, IOM 312F-98-483

Tokumaru, M., Kojima, M., \& Fujiki, K. 2010, J. Geophys. Res. (Space Physics), 115,4102

Woo, R., \& Habbal, S. R. 1999, Geophys. Res. Lett., 26, 1793

You, X. P., Hobbs, G. B., Coles, W. A., Manchester, R. N., \& Han, J. L. 2007, ApJ, 671, 907

You, X. P., Coles, W. A., Hobbs, G. B., \& Manchester, R. N. 2012, MNRAS, 422, 1160

Zielenbach, W. 2011, ApJ, 142, 120 
Table 5. Masses of the 59 asteroids that induce perturbations greater than $5 \mathrm{~m}$ on the Earth-Mars distances during the period of observations (Kuchynka et al. 2010).

\begin{tabular}{|c|c|c|c|c|c|c|c|}
\hline $\begin{array}{l}\text { IAU designation } \\
\text { number }\end{array}$ & $\begin{array}{l}\text { INPOP10c } \\
10^{12} \times M_{\odot}\end{array}$ & $\begin{array}{l}\text { INPOP10d } \\
10^{12} \times M_{\odot}\end{array}$ & $\begin{array}{c}\text { Diff } \\
10^{12} \times M_{\odot}\end{array}$ & S & $\underset{\mathrm{m}}{\text { Impact }}$ & $\begin{array}{l}L \\
\%\end{array}$ & \\
\hline 4 & $130.109 \pm 0.716$ & $130.109 \pm 0.983$ & 0.000 & & 1198.953 & 20.5 & $\mathrm{~S}$ \\
\hline 1 & $467.267 \pm 2.047$ & $467.267 \pm 2.437$ & 0.000 & & 793.741 & 17.3 & $\mathrm{~S}$ \\
\hline 2 & $103.843 \pm 1.689$ & $102.654 \pm 1.933$ & 1.189 & & 146.270 & 11.8 & $\mathrm{~S}$ \\
\hline 324 & $5.723 \pm 0.531$ & $5.723 \pm 0.611$ & 0.000 & & 93.536 & 1.0 & S \\
\hline 10 & $43.513 \pm 3.300$ & $43.513 \pm 3.877$ & 0.000 & & 77.003 & 15.9 & $\mathrm{~S}$ \\
\hline 19 & $3.884 \pm 0.447$ & $3.450 \pm 0.526$ & 0.435 & & 59.069 & 13.8 & $\mathrm{~N}$ \\
\hline 3 & $11.793 \pm 0.714$ & $11.793 \pm 0.803$ & 0.000 & & 55.639 & 0.6 & $\mathrm{~S}$ \\
\hline 704 & $19.217 \pm 2.315$ & $19.217 \pm 2.869$ & 0.000 & & 34.492 & 7.4 & $\mathrm{~S}$ \\
\hline 532 & $2.895 \pm 1.043$ & $2.895 \pm 1.093$ & 0.000 & & 32.714 & 2.3 & $\mathrm{~S}$ \\
\hline 9 & $3.864 \pm 0.613$ & $3.063 \pm 0.665$ & 0.801 & & 29.606 & 20.6 & $\mathrm{~N}$ \\
\hline 7 & $5.671 \pm 0.512$ & $5.367 \pm 0.591$ & 0.305 & & 27.822 & 13.9 & $\mathrm{~S}$ \\
\hline 29 & $7.629 \pm 1.067$ & $7.227 \pm 1.225$ & 0.402 & & 26.673 & 2.9 & $\mathrm{~S}$ \\
\hline 24 & $7.641 \pm 1.596$ & $2.194 \pm 1.775$ & 5.447 & $*$ & 26.131 & 36.0 & $\mathrm{~N}$ \\
\hline 31 & $3.256 \pm 2.034$ & $4.411 \pm 2.050$ & 1.155 & & 23.466 & 24.1 & $\mathrm{~S}$ \\
\hline 15 & $13.576 \pm 0.939$ & $13.576 \pm 1.264$ & 0.000 & & 21.555 & 20.6 & $\mathrm{~S}$ \\
\hline 6 & $7.084 \pm 0.822$ & $7.084 \pm 1.048$ & 0.000 & & 21.150 & 7.4 & S \\
\hline 11 & $3.771 \pm 0.976$ & $3.771 \pm 1.110$ & 0.000 & & 17.301 & 31.9 & S \\
\hline 139 & $0.000 \pm 0.000$ & $3.579 \pm 0.595$ & 3.579 & $\mathrm{~N}$ & 16.687 & 32.0 & $\mathrm{~N}$ \\
\hline 747 & $4.129 \pm 0.841$ & $6.805 \pm 1.089$ & 2.676 & $*$ & 15.937 & 31.6 & $\mathrm{~N}$ \\
\hline 105 & $3.111 \pm 0.556$ & $3.111 \pm 0.745$ & 0.000 & & 15.196 & 4.5 & $\mathrm{~N}$ \\
\hline 20 & $0.000 \pm 0.000$ & $1.921 \pm 0.661$ & 1.921 & $\mathrm{~N}$ & 14.763 & 39.9 & $\mathrm{~N}$ \\
\hline 372 & $12.365 \pm 2.676$ & $12.365 \pm 2.990$ & 0.000 & & 13.796 & 19.0 & $\mathrm{~S}$ \\
\hline 8 & $3.165 \pm 0.353$ & $3.325 \pm 0.365$ & 0.159 & & 12.664 & 17.7 & $\mathrm{~S}$ \\
\hline 45 & $3.523 \pm 0.819$ & $1.518 \pm 0.962$ & 2.005 & $*$ & 11.790 & 21.0 & $\mathrm{~N}$ \\
\hline 41 & $3.836 \pm 0.721$ & $2.773 \pm 0.977$ & 1.063 & & 11.568 & 15.2 & $\mathrm{~N}$ \\
\hline 405 & $0.005 \pm 0.003$ & $0.006 \pm 0.003$ & 0.001 & & 11.378 & 21.2 & $\mathrm{~N}$ \\
\hline 511 & $9.125 \pm 2.796$ & $9.125 \pm 3.138$ & 0.000 & & 10.248 & 20.5 & S \\
\hline 52 & $8.990 \pm 2.781$ & $8.990 \pm 3.231$ & 0.000 & & 9.841 & 3.0 & S \\
\hline 16 & $12.613 \pm 2.286$ & $12.613 \pm 2.746$ & 0.000 & & 9.701 & 8.9 & $\mathrm{~S}$ \\
\hline 419 & $1.185 \pm 0.461$ & $0.425 \pm 0.398$ & 0.760 & & 9.585 & 10.2 & $\mathrm{~N}$ \\
\hline 78 & $0.026 \pm 0.016$ & $0.024 \pm 0.016$ & 0.002 & & 9.389 & 9.8 & $\mathrm{~N}$ \\
\hline 259 & $0.092 \pm 0.002$ & $0.006 \pm 0.003$ & 0.086 & & 9.222 & 31.4 & $\mathrm{~N}$ \\
\hline 27 & $0.000 \pm 0.000$ & $1.511 \pm 0.982$ & 1.511 & $\mathrm{~N}$ & 9.146 & 29.5 & $\mathrm{~N}$ \\
\hline 23 & $0.000 \pm 0.000$ & $0.093 \pm 0.156$ & 0.093 & $\mathrm{~N}$ & 9.067 & 31.1 & $\mathrm{~N}$ \\
\hline 488 & $3.338 \pm 1.850$ & $0.000 \pm 0.000$ & 3.338 & 0 & 8.614 & 2.8 & $\mathrm{~N}$ \\
\hline 230 & $0.000 \pm 0.000$ & $0.263 \pm 0.169$ & 0.263 & $\mathrm{~N}$ & 7.620 & 27.0 & $\mathrm{~N}$ \\
\hline 409 & $0.002 \pm 0.001$ & $0.002 \pm 0.001$ & 0.000 & 0 & 7.574 & 2.2 & $\mathrm{~N}$ \\
\hline 94 & $1.572 \pm 1.097$ & $7.631 \pm 2.488$ & 6.058 & $*$ & 7.466 & 28.5 & $\mathrm{~N}$ \\
\hline 344 & $2.701 \pm 0.497$ & $2.088 \pm 0.515$ & 0.613 & & 7.465 & 15.3 & $\mathrm{~N}$ \\
\hline 130 & $0.099 \pm 0.047$ & $0.221 \pm 0.069$ & 0.122 & $*$ & 7.054 & 31.7 & $\mathrm{~N}$ \\
\hline 111 & $1.002 \pm 0.323$ & $0.000 \pm 0.000$ & 1.002 & 0 & 6.985 & 11.4 & $\mathrm{~N}$ \\
\hline 109 & $0.495 \pm 0.322$ & $1.318 \pm 0.852$ & 0.823 & & 6.865 & 18.7 & $\mathrm{~N}$ \\
\hline 42 & $1.144 \pm 0.362$ & $0.083 \pm 0.389$ & 1.061 & $*$ & 6.829 & 0.6 & $\mathrm{~N}$ \\
\hline 63 & $0.000 \pm 0.000$ & $0.424 \pm 0.143$ & 0.424 & $\mathrm{~N}$ & 6.451 & 17.4 & $\mathrm{~N}$ \\
\hline 12 & $2.297 \pm 0.319$ & $1.505 \pm 0.331$ & 0.792 & $*$ & 6.159 & 21.7 & $\mathrm{~N}$ \\
\hline 469 & $0.088 \pm 0.073$ & $0.000 \pm 0.000$ & 0.088 & 0 & 6.107 & 18.1 & $\mathrm{~N}$ \\
\hline 144 & $0.176 \pm 0.297$ & $0.751 \pm 0.361$ & 0.575 & & 6.087 & 22.8 & $\mathrm{~N}$ \\
\hline
\end{tabular}

Notes. Columns 2 and 3 give the values of the masses with the 1- $\sigma$ uncertainties obtained with INPOP10c and INPOP10d. The differences between INPOP10c and INPOP10d values are given in Col. 4. Column 5 indicates if the INPOP10d masses are newly determined (quoted N), set equal to 0 when estimated by INPOP10c (quoted 0), significantly different from INPOP10c determinations $(*)$. The maximum impacts of the perturbations are given in Col. 5. In Col. 7 is given the $L$ criteria, the rate of local maxima rejected from the INPOP10c fit but included in the INPOP10d. In the last column we provide the status of the imposed constraints: $\mathrm{S}$ for strong and $\mathrm{N}$ for normal. 
A. K. Verma et al.: Electron density distribution from MGS, MEX, and VEX

Table 5. continued.

\begin{tabular}{lccccccc}
\hline \hline $\begin{array}{l}\text { IAU designation } \\
\text { number }\end{array}$ & $\begin{array}{c}\text { INPOP10c } \\
10^{12} \times M_{\odot}\end{array}$ & $\begin{array}{c}\text { INPOP10d } \\
10^{12} \times M_{\odot}\end{array}$ & $\begin{array}{c}\text { Diff } \\
10^{12} \times M_{\odot}\end{array}$ & $\begin{array}{c}\text { Impact } \\
\mathrm{m}\end{array}$ & $\begin{array}{c}\mathrm{L} \\
\%\end{array}$ \\
\hline 356 & $4.173 \pm 0.868$ & $4.173 \pm 0.902$ & 0.000 & & 5.759 & 2.2 & $\mathrm{~N}$ \\
712 & $0.000 \pm 0.000$ & $1.228 \pm 0.267$ & 1.228 & $\mathrm{~N}$ & 5.745 & 2.2 & $\mathrm{~N}$ \\
88 & $1.340 \pm 0.866$ & $0.000 \pm 0.000$ & 1.340 & 0 & 5.742 & 1.4 & $\mathrm{~N}$ \\
60 & $0.402 \pm 0.221$ & $0.282 \pm 0.268$ & 0.120 & & 5.733 & 3.8 & $\mathrm{~N}$ \\
50 & $0.686 \pm 0.187$ & $1.031 \pm 0.566$ & 0.345 & & 5.702 & 2.0 & $\mathrm{~N}$ \\
128 & $4.699 \pm 1.522$ & $0.000 \pm 0.000$ & 4.677 & 0 & 5.624 & 3.0 & $\mathrm{~N}$ \\
5 & $0.448 \pm 0.165$ & $0.913 \pm 0.220$ & 0.466 & $*$ & 5.533 & 15.8 & $\mathrm{~N}$ \\
59 & $4.332 \pm 0.607$ & $1.364 \pm 1.097$ & 2.968 & $*$ & 5.325 & 12.1 & $\mathrm{~N}$ \\
98 & $1.414 \pm 0.603$ & $2.100 \pm 0.705$ & 0.686 & & 5.195 & 15.1 & $\mathrm{~N}$ \\
194 & $6.387 \pm 0.701$ & $4.380 \pm 0.819$ & 2.007 & $*$ & 5.145 & 2.9 & $\mathrm{~N}$ \\
51 & $3.546 \pm 0.748$ & $3.639 \pm 0.937$ & 0.093 & & 5.109 & 15.6 & $\mathrm{~N}$ \\
156 & $3.263 \pm 0.438$ & $3.089 \pm 0.576$ & 0.174 & & 5.103 & 19.3 & $\mathrm{~N}$ \\
\hline
\end{tabular}

Table 6. Asteroid masses found in the recent literature compared with the values estimated in INPOP10c and INPOP10d.

\begin{tabular}{|c|c|c|c|c|c|}
\hline $\begin{array}{l}\text { IAU designation } \\
\text { number }\end{array}$ & $\begin{array}{l}\text { INPOP10c } \\
10^{12} \times M_{\odot}\end{array}$ & $\begin{array}{c}\text { Close-encounters } \\
10^{12} \times M_{\odot}\end{array}$ & Refs. & $\begin{array}{l}\text { INPOP10d } \\
10^{12} \times M_{\odot} \\
\end{array}$ & $\%$ \\
\hline 5 & $0.448 \pm 0.165$ & $1.705 \pm 0.348$ & Zielenbach (2011) & $0.913 \pm 0.220$ & 15.8 \\
\hline 12 & $2.297 \pm 0.319$ & $2.256 \pm 1.910$ & Zielenbach (2011) & $1.505 \pm 0.331$ & 21.7 \\
\hline 20 & $0.000 \pm 0.000$ & $1.680 \pm 0.350$ & Baer et al. (2011) & $1.921 \pm 0.661$ & 39.9 \\
\hline 24 & $7.6 \pm 1.6$ & $2.639 \pm 1.117$ & Zielenbach (2011) & $2.2 \pm 1.7$ & 36.0 \\
\hline 27 & $0.000 \pm 0.000$ & $1.104 \pm 0.732$ & Zielenbach (2011) & $1.511 \pm 0.982$ & 29.5 \\
\hline 45 & $3.523 \pm 0.819$ & $2.860 \pm 0.060$ & Marchis et al. (2008) & $1.518 \pm 0.962$ & 21.0 \\
\hline 59 & $4.332 \pm 0.607$ & $1.448 \pm 0.0187$ & Zielenbach (2011) & $1.364 \pm 1.097$ & 12.1 \\
\hline 94 & $1.572 \pm 1.097$ & $7.878 \pm 4.016$ & Zielenbach (2011) & $7.631 \pm 2.488$ & 28.5 \\
\hline 130 & $0.099 \pm 0.047$ & $3.320 \pm 0.200$ & Marchis et al. (2008) & $0.221 \pm 0.069$ & 31.7 \\
\hline 139 & $0.000 \pm 0.000$ & $3.953 \pm 2.429$ & Zielenbach (2011) & $3.579 \pm 0.595$ & 32.0 \\
\hline
\end{tabular}

Notes. The uncertainties are given at 1 published sigma. 


\section{Appendix A: Analytical solution}

This section presents the analytical solutions of Eq. (1). Let, $I_{1}$ and $I_{2}$ be the integral solutions of Eq. (1) using Eqs. (3) and (4) i.e.,

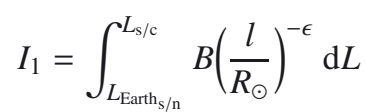

and

$I_{2}=\int_{L_{\mathrm{Earth} / \mathrm{n}}}^{L_{\mathrm{s} / \mathrm{c}}}\left[A\left(\frac{l}{R_{\odot}}\right)^{-4}+B\left(\frac{l}{R_{\odot}}\right)^{-2}\right] \mathrm{d} L$.

From the geometry (Fig. 3) we define

$P=R_{\mathrm{S} / \mathrm{E}} \sin \alpha$,

$L_{\mathrm{DC}}=R_{\mathrm{E} / \mathrm{SC}}-R_{\mathrm{S} / \mathrm{E}} \cos \alpha$,

$L_{\mathrm{DE}}^{2}=l^{2}-P^{2}$,

$l^{2}=L^{2}+R_{\mathrm{S} / \mathrm{E}}^{2}-2 L R_{\mathrm{S} / \mathrm{E}} \cos \alpha$,

where $\alpha$ and $\beta$ are the angle between the Sun-Earth-Probe (SEP) and the Earth-Sun-Probe (ESP). $P$ is the MDLOS from the Sun. With these expressions, $I_{1}$ can be written as

$$
\begin{aligned}
I_{1} & =\int_{L_{\mathrm{Earrh}} / \mathrm{n}}^{L_{\mathrm{s} / \mathrm{c}}} B R_{\odot}^{\epsilon}\left(\frac{\mathrm{d} L}{\left(L^{2}+R_{\mathrm{S} / \mathrm{E}}^{2}-2 L R_{\mathrm{S} / \mathrm{E}} \cos \alpha\right)^{\epsilon / 2}}\right) \\
& =B R_{\odot}^{\epsilon} \int_{L_{\mathrm{Earth} / \mathrm{n}}}^{L_{\mathrm{s} / \mathrm{c}}}\left(\frac{\mathrm{d} L}{\left(\left[L-R_{\mathrm{S} / \mathrm{E}} \cos \alpha\right]^{2}+R_{\mathrm{S} / \mathrm{E}}^{2} \sin ^{2} \alpha\right)^{\epsilon / 2}}\right) .
\end{aligned}
$$

Assuming,

$x=L-R_{\mathrm{S} / \mathrm{E}} \cos \alpha$,

$a=R_{\mathrm{S} / \mathrm{E}} \sin \alpha$,

$\mathrm{d} x=\mathrm{d} L$,

with $L=0$ at the Earth station $\left(L_{\mathrm{Earth}} / \mathrm{n}\right)$ and $L=R_{\mathrm{E} / \mathrm{SC}}$ at the spacecraft $\left(L_{\mathrm{S} / \mathrm{c}}\right)$. Then the integral $I_{1}$ can be written as

$$
\begin{aligned}
I_{1} & =B R_{\odot}^{\epsilon} \int_{-R_{\mathrm{S} / \mathrm{E}} \cos \alpha}^{R_{\mathrm{E} / \mathrm{CC}}-R_{\mathrm{S} / \mathrm{E}} \cos \alpha} \frac{\mathrm{d} x}{\left(x^{2}+a^{2}\right)^{\epsilon / 2}} \\
& =\frac{B R_{\odot}^{\epsilon}}{a^{\epsilon}} \int_{-R_{\mathrm{S} / \mathrm{E}} \cos \alpha}^{R_{\mathrm{E} / \mathrm{SC}}-R_{\mathrm{S} / \mathrm{E}} \cos \alpha} \frac{\mathrm{d} x}{\left(1+\frac{x^{2}}{a^{2}}\right)^{\epsilon / 2}} .
\end{aligned}
$$

Now let

$\frac{x}{a}=\tan \theta$,

and

$\mathrm{d} x=a \sec ^{2} \theta \mathrm{d} \theta$.

Therefore,

$I_{1}=\frac{B R_{\odot}^{\epsilon}}{a^{\epsilon}} \int_{\arctan \left(\frac{\left(-R_{\mathrm{S} / \mathrm{E}} \cos \alpha\right)}{a}\right)}^{\arctan \left(\frac{\left(R_{\mathrm{E} / S \mathrm{C}}-R_{\mathrm{S} / \mathrm{E}} \cos \alpha\right)}{a}\right)} \frac{a \sec ^{2} \theta}{\left(\tan ^{2} \theta+1\right)^{\epsilon / 2}} \mathrm{~d} \theta$.

From the geometry of Fig. 3, the lower limit of Eq. (A.3) can be written as

$\arctan \left(\frac{-R_{\mathrm{S} / \mathrm{E}} \cos \alpha}{a}\right)=\arctan \left(\frac{-R_{\mathrm{S} / \mathrm{E}} \cos \alpha}{R_{\mathrm{S} / \mathrm{E}} \sin \alpha}\right)$,

A124, page 16 of 17 with

$\cot \alpha=\tan \left(\frac{\pi}{2}-\alpha\right)$.

Hence,

$\arctan \left(\frac{-R_{\mathrm{S} / \mathrm{E}} \cos \alpha}{a}\right)=\alpha-\frac{\pi}{2}$.

Similarly, the upper limit of Eq. (A.3) can be written as

$\arctan \left(\frac{R_{\mathrm{E} / \mathrm{SC}}-R_{\mathrm{S} / \mathrm{E}} \cos \alpha}{a}\right)=\arctan \left(\frac{R_{\mathrm{E} / \mathrm{SC}}-R_{\mathrm{S} / \mathrm{E}} \cos \alpha}{R_{\mathrm{S} / \mathrm{E}} \sin \alpha}\right)$,

with

$R_{\mathrm{E} / \mathrm{SC}}-R_{\mathrm{S} / \mathrm{E}} \cos \alpha=R_{\mathrm{S} / \mathrm{E}} \sin \alpha\left[\tan \left(\beta-\left\{\frac{\pi}{2}-\alpha\right\}\right)\right]$.

Hence,

$\arctan \left(\frac{R_{\mathrm{E} / \mathrm{SC}}-R_{\mathrm{S} / \mathrm{E}} \cos \alpha}{a}\right)=\beta+\alpha-\frac{\pi}{2}$.

Now Eq. (A.3) is given by

$I_{1}=\frac{B R_{\odot}^{\epsilon}}{a^{\epsilon}} \int_{\alpha-\frac{\pi}{2}}^{\beta+\alpha-\frac{\pi}{2}} \frac{a \sec ^{2} \theta}{\left(\tan ^{2} \theta+1\right)^{\epsilon / 2}} \mathrm{~d} \theta$.

with

$\sec ^{2} \theta=\tan ^{2} \theta+1$,

and

$\cos \theta=\frac{1}{\sec \theta}$.

Therefore, the integral $I_{1}$ can be written as

$I_{1}=\frac{B R_{\odot}^{\epsilon}}{a^{\epsilon-1}} \int_{\alpha-\frac{\pi}{2}}^{\beta+\alpha-\frac{\pi}{2}}(\cos \theta)^{\epsilon-2} \mathrm{~d} \theta$.

The maximum contribution of the integral occurs at $\theta=0$. To solve this integral, Taylor series expansion was used and for $\theta$ near zero, it can be given as

$$
\begin{aligned}
f(\theta)= & f(0)+\theta\left(\frac{\mathrm{d} f}{\mathrm{~d} \theta}\right)+\frac{\theta^{2}}{2 !}\left(\frac{\mathrm{d}^{2} f}{\mathrm{~d} \theta^{2}}\right)+\frac{\theta^{3}}{3 !}\left(\frac{d^{3} f}{d \theta^{3}}\right) \\
& +\frac{\theta^{4}}{4 !}\left(\frac{\mathrm{d}^{4} f}{\mathrm{~d} \theta^{4}}\right) \ldots+\theta\left(\theta^{n}\right),
\end{aligned}
$$

with

$f(\theta)=(\cos \theta)^{\epsilon-2}$.

Then

$f(0)=1$

$\left(\frac{\mathrm{d} f}{\mathrm{~d} \theta}\right)=-(\epsilon-2)(\sin \theta)(\cos \theta)^{(\epsilon-3)}$,

$\left(\frac{\mathrm{d} f}{\mathrm{~d} \theta}\right)_{\theta=0}=0$,

$\left(\frac{\mathrm{d}^{2} f}{\mathrm{~d} \theta^{2}}\right)=(\epsilon-2)(\epsilon-3)\left(\sin ^{2} \theta\right)(\cos \theta)^{(\epsilon-4)}$ $-(\epsilon-2)(\cos \theta)^{(\epsilon-2)}$, 
A. K. Verma et al.: Electron density distribution from MGS, MEX, and VEX

$\left(\frac{\mathrm{d}^{2} f}{\mathrm{~d} \theta^{2}}\right)_{\theta=0}=-(\epsilon-2)$,

$\left(\frac{\mathrm{d}^{3} f}{\mathrm{~d} \theta^{3}}\right)=-(\epsilon-2)(\epsilon-3)(\epsilon-4)\left(\sin ^{3} \theta\right)(\cos \theta)^{(\epsilon-5)}$

$$
+(\epsilon-2)(3 \epsilon-8)(\sin \theta)(\cos \theta)^{(\epsilon-3)},
$$

$\left(\frac{\mathrm{d}^{3} f}{\mathrm{~d} \theta^{3}}\right)_{\theta=0}=0$,

$\left(\frac{\mathrm{d}^{4} f}{\mathrm{~d} \theta^{4}}\right)=(\epsilon-2)(\epsilon-3)(\epsilon-4)(\epsilon-5)\left(\sin ^{4} \theta\right)(\cos \theta)^{(\epsilon-6)}$

$$
-(\epsilon-2)(\epsilon-3)(6 \epsilon-20)\left(\sin ^{2} \theta\right)(\cos \theta)^{(\epsilon-4)}
$$$$
+(\epsilon-2)(3 \epsilon-8)(\cos \theta)^{(\epsilon-2)} \text {, }
$$

$\left(\frac{\mathrm{d}^{4} f}{\mathrm{~d} \theta^{4}}\right)_{\theta=0}=(\epsilon-2)(3 \epsilon-8)$.

Now, Eq. (A.5) can be written as

$$
\begin{aligned}
(\cos \theta)^{(\epsilon-2)} & =1-(\epsilon-2) \frac{\theta^{2}}{2 !}+(\epsilon-2)(3 \epsilon-8) \frac{\theta^{4}}{4 !}+\ldots \theta\left(\theta^{n}\right) \\
& =1-\frac{\epsilon-2}{2} \theta^{2}+\frac{3 \epsilon^{2}-14 \epsilon+16}{24} \theta^{4}+\ldots \theta\left(\theta^{n}\right) .
\end{aligned}
$$

By neglecting the higher order terms, the integral (Eq. (A.4)) can be written as

$$
\begin{aligned}
I_{1}= & \frac{B R_{\odot}^{\epsilon}}{a^{\epsilon-1}} \int_{\alpha-\frac{\pi}{2}}^{\beta+\alpha-\frac{\pi}{2}}\left(1-\frac{\epsilon-2}{2} \theta^{2}+\frac{3 \epsilon^{2}-14 \epsilon+16}{24} \theta^{4}\right) \mathrm{d} \theta \\
= & \frac{B R_{\odot}^{\epsilon}}{a^{\epsilon-1}}\left[\theta-\frac{\epsilon-2}{6} \theta^{3}+\frac{3 \epsilon^{2}-14 \epsilon+16}{120} \theta^{5}\right]_{\alpha-\frac{\pi}{2}}^{\beta+\alpha-\frac{\pi}{2}} \\
= & \frac{B R_{\odot}^{\epsilon}}{a^{\epsilon-1}}\left[\beta-\frac{\epsilon-2}{6}\left((\beta+\alpha-\pi / 2)^{3}-(\alpha-\pi / 2)^{3}\right)\right. \\
& \left.+\frac{3 \epsilon^{2}-14 \epsilon+16}{120}\left((\beta+\alpha-\pi / 2)^{5}-(\alpha-\pi / 2)^{5}\right)\right] .
\end{aligned}
$$

By substituting the value of $a$, we can write the integral $I_{1}$ as

$$
\begin{aligned}
I_{1}= & \frac{B R_{\odot}^{\epsilon}}{\left(R_{\mathrm{S} / \mathrm{E}} \sin \alpha\right)^{\epsilon-1}}\left[\beta-\frac{\epsilon-2}{6}\left((\beta+\alpha-\pi / 2)^{3}-(\alpha-\pi / 2)^{3}\right)\right. \\
& \left.+\frac{3 \epsilon^{2}-14 \epsilon+16}{120}\left((\beta+\alpha-\pi / 2)^{5}-(\alpha-\pi / 2)^{5}\right)\right] .
\end{aligned}
$$

Now, Eq. (A.2) can be written as $I_{2}=I_{2 a}+I_{2 b}$ where,

$$
I_{2 a}=\int_{L_{\mathrm{Earth}} / \mathrm{n}}^{L_{\mathrm{s} / \mathrm{c}}} A\left(\frac{l}{R_{\odot}}\right)^{-4} \mathrm{~d} L,
$$

and

$I_{2 b}=\int_{L_{\mathrm{Earth}} / \mathrm{n}}^{L_{\mathrm{s} / \mathrm{c}}} B\left(\frac{l}{R_{\odot}}\right)^{-2} \mathrm{~d} L$.

Using a similar approach to the previous integral, one can write

$$
\begin{aligned}
I_{2 a} & =\frac{A R_{\odot}^{4}}{a^{3}} \int_{\alpha-\frac{\pi}{2}}^{\beta+\alpha-\frac{\pi}{2}} \cos ^{2} \theta \mathrm{d} \theta \\
& =\frac{A R_{\odot}^{4}}{a^{3}}\left[\frac{1}{4}(2 \theta+\sin 2 \theta)\right]_{\alpha-\frac{\pi}{2}}^{\beta+\alpha-\frac{\pi}{2}} \\
& =\frac{A R_{\odot}^{4}}{4 a^{3}}[(2 \beta-\sin 2(\alpha+\beta)+\sin 2 \alpha)] \\
& =\frac{A R_{\odot}^{4}}{4 R_{\mathrm{S} / \mathrm{E}}^{3} \sin ^{3} \alpha}[(2 \beta-\sin 2(\alpha+\beta)+\sin 2 \alpha)] .
\end{aligned}
$$

Similarly, Eq. (A.8) can be written as

$$
\begin{aligned}
I_{2 b} & =\frac{B R_{\odot}^{2}}{a} \int_{\alpha-\frac{\pi}{2}}^{\beta+\alpha-\frac{\pi}{2}} \mathrm{~d} \theta \\
& =\frac{B R_{\odot}^{2}}{a}[\theta]_{\alpha-\frac{\pi}{2}}^{\beta+\alpha-\frac{\pi}{2}} \\
& =\frac{B R_{\odot}^{2}}{R_{\mathrm{S} / \mathrm{E}} \sin \alpha} \beta .
\end{aligned}
$$

Now by using $I_{2 a}$ and $I_{2 b}$, expression $I_{2}$ can be written as

$$
\begin{aligned}
I_{2}= & \frac{A R_{\odot}^{4}}{4 R_{\mathrm{S} / \mathrm{E}}^{3} \sin ^{3} \alpha}[(2 \beta-\sin 2(\alpha+\beta)+\sin 2 \alpha)] \\
& +\frac{B R_{\odot}^{2}}{R_{\mathrm{S} / \mathrm{E}} \sin \alpha} \beta .
\end{aligned}
$$

